\title{
Phytosociological remarks on residual woodlands of Laurus nobilis in Sicily
}

\author{
Lorenzo Gianguzzi, Agostino D'Amico \& Salvatore Romano (*)
}

\begin{abstract}
Gianguzzi L., D'Amico, A. \& Romano, S. Phytosociological remarks on residual woodlands of Laurus nobilis in Sicily. Lazaroa 31: 67-84 (2010).

A phytosociological study was done of Laurus nobilis woodlands in Sicily. The analysis, based on 18 relevés, supports the definition of a new syntaxon (Acantho mollis-Lauretum nobilis ass. nov.), considered as a vicariant of similar vegetation aspects described in other Mediterranean areas (Iberian and Italian peninsulas). The study mainly includes some unpublished sites where polycormic individuals of laurel, sometimes reaching 13-15 meters in height, dominates the tree layer. This vegetation is also characterized by some laurophyllous species (Hedera helix, Rhamnus alaternus, Smilax aspera and sometimes Viburnum tinus) and lianas (Rubia peregrina var. longifolia, Asparagus acutifolius, Tamus communis, Clematis vitalba, Calystegia sylvatica and Rubus ulmifolius), while in the undergrowth comprises particularly various broadleaved elements such as Cyclamen hederifolium subsp. confusum, and Acanthus mollis subsp. mollis, both included in the characteristic species combination together with Pistacia terebinthus and Orobanche hederae. The coenosis is typical of the humic soils, often rich in lithic components, of shaded ravines and peririparian environments, in the thermo- and mesomediterranean thermotype (Mediterranean pluviseasonal oceanic bioclimate). In view of the relictual status of this vegetation - also included as a "Priority Habitat" in Council Directive 92/43/EEC - we underline the need for a suitable protection and conservation of these small, fragmented and isolated patches.
\end{abstract}

Keywords: Laurus nobilis matorral, Quercetea ilicis, Mediterranean region, relic vegetation.

Resumen: Gianguzzi L., D'Amico, A. \& Romano, S. Estudio fitosociológico de las comunidades de Laurus nobilis en Sicilia Lazaroa 31: 67-84 (2010).

Se ha realizado un estudio fitosociológico sobre los bosques de Laurus nobilis en Sicilia. El análisis, basado en 18 inventarios nos ha servido para la definición de un nuevo sintaxon (Acantho mollis-Lauretum nobilis nova), que puede ser considerado como un vicariante de tipos de vegetación similares descritos en otras áreas mediterráneas (Península Ibérica e Itálica). El estudio incluye el muestreo de sitios inéditos donde los individuos de laurel alcanzan hasta 13-15 metros de altura y dominan el estrato arbóreo. Este tipo de vegetación también está caracterizado por otras especies lauroides (Hedera helix, Rhamnus alaternus, Smilax aspera y, a veces, Viburnum tinus) y lianas (Rubia peregrina var. longifolia, Asparagus acutifolius, Tamus communis, Clematis vitalba, Calystegia sylvatica y Rubus ulmifolius), mientras el estrato arbustivo comprende varios elementos de hoja ancha como Cyclamen hederifolium subsp. confusum, y Acanthus mollis, junto a Pistacia terebinthus u Orobanche hederae. Esta comunidad es típica de los suelos húmicos, a menudo pedregosos y propio de barrancos sombríos y medios peri-riparios, en los pisos bioclimáticos termo-y mesomediterráneo (Mediterráneo pluviestacional oceánico). A la vista del estado reliquial de este tipo de bosque, incluido como un "Hábitat Prioritario" en el Consejo la Directiva 92/43/EEC, subrayamos la necesidad de definir la protección más conveniente y la conservación de estos pequeños, fragmentados y aislados bosquetes.

Palabras clave: bosquetes de Laurus nobilis, Quercetea ilicis, Región Mediterránea, vegetación relíctica.

\section{INTRODUCTION}

Laurus nobilis L. (bay laurel), together with $L$. azorica (Seub.) Franco, and L. novocanariensis
Rivas Mart., Louŝa, Fern. Prieto, E. Días, J. C. Costa \& Aguiar (= L. canariensis Webb \& Berthel.), is one of the three known species of the genus and also the only representative of Laura-

\footnotetext{
* Department of Botanical Science. via Archirafi 38. 90123 Palermo. Italy
} 
ceae in continental Europe - a family including about 2500 taxa distributed in tropical and subtropical areas (Ferguson, 1974; HeYwood, 1993; MabBerLey, 1997). Given that the other two species are located in the Azores, with isolated sites in Morocco (BARBERO \& QUEZEL, 1994; and as recently revealed (RIVAS-MARTíNEZ \& al., 2002), in the Canary Islands and Madeira (JALAS \& SuOminen, 1991; Tutin \& al., 1993).

L. nobilis occurs in different areas of the Mediterranean basin, such as in the south-east France, Italy, ex-Yugoslavia, Albania, Greece, Turkey, Cyprus, Lebanon, Israel, Cyrenaica, Algeria, Morocco, Sicily, Sardinia, Corsica and the Balearic Island (Meusel \& al., 1965; FenAROLI \& GAMBi, 1976; JALAS \& SUOMINEN, 1991). The species also occurs in other areas of the Eurosiberian Region, as along the Black Sea basin (Komarov, 1937; Meusel \& al., 1965; FenAROLI \& GAMBI, 1976), the Atlantic coasts of the Iberian peninsula, northern France, southern England and Ireland (JaLAS \& SuOMinen, 1991; STACE, 1991). Nevertheless, because of its ancient cultivation - dating back at least to the Roman period - and its out-standing tendency to naturalize, it is essential to identify its indigenous geographical areas, in view of the lack of molecular studies, in order to clarify phyletic relationships among different populations (FILIBECK, 2006).

Bay laurel is typical of thermo-hygrophilous coenosis of Mediterranean areas (Table 1), mainly represented in pre-forest mantles or woodlands, where it appears frequently as a sporadic shrub species. More rare are the vegetation types in which L. nobilis shows a tree habitus, which can probably be interpreted as relictual fragments of a Laurinee thermo-hygrophilous vegetation widely represented in continental Europe during the Tertiary and the warm periods at the beginning of the Quaternary (MONTELUCCI, 1946; GIACOMINI \& Fenaroli, 1958; Pignatti, 1976; 1995). As a consequence of climate change during the Pleistocene, most of the dominant species became extinct, while others, like L. nobilis, found refugia in some new communities which colonized the region. As a result, some laurophyllous vegetation aspects, with an impoverished floristic composi- tion, became isolated along the Atlantic coast of Europe or in the extreme southern part of the continent. This is the case of the formation of communities dominated by Laurus nobilis on the Iberian peninsula (BUENO SÁNCHEZ \& FERNÁNDEZ Prieto, 1991; RodríGuez Guitían \& al., 2007), or fragmentarily represented in the Apennines area (ALLEGREZZA \& al., 2006), as well as in Sardinia (BACCHETTA \& al., 2007) and Sicily, where they were known in the Madonie (RAIMONDO \& al., 1980) and Hyblaean mountains (BRULLO \& al., 2001).

Some interesting new discoveries of Laurus nobilis woodlands in central-western Sicily gave rise to a regional phytosociological analysis of this vegetation, and a subsequent comparison to other similar aspects known from the Mediterranean area. The research considered particularly those phytocoenotic expressions where bay laurel has a strong dominant role, or where it definitely characterizes the physiognomy of the tree layer.

\section{DISTRIBUTION OF LAURUS NOBILIS IN SICILY}

In Sicily Laurus nobilis shows a very scattered distribution, while it is absent from the small circum-Sicilian islets. GuSSONE (1842-44) indicated its presence in Lampedusa Island (in Vallone dello Scoglio), where it became extinct, probably due to the strong anthropization during last century (BARTOLO \& al., 1990b; PASTA, 2001). LOJACONO (1904) considered bay laurel in the region to be an element that was "...rather cultivated than decidedly indigenous ..." an opinion also shared by Albo (1919) and BÉGUINOT (1929). More recently, other authors agreed on the indigenous status of the species in the region (RAIMONDO \& al., 1980; BRUllo \& al., 2001; GianguzZi \& al., 2007).

In Sicily, vegetation aspects in which L. nobilis plays a relevant role have been cited for different sites in: -Mt. Cammarata (BonOMO \& al., 1978); -Madonie, near the Vicaretto stream (RAIMONDO \& al., 1980); -Peloritani, in Vallone della Santissima (BARTOLO \& al., 1990) and Valloncello Passo del Corvo, between Borgo Morfia and Portella Mandrazzi (GuARINO, 1998); -the hills 
Table 1

Aspects of vegetation with Laurus nobilis known for Mediterranean Region.

\section{A) SCLEROPHYLL EVERGREEN WOODLANDS}

\begin{tabular}{|c|c|}
\hline Quercus ilex & $\begin{array}{l}\text { Italian peninsula (PAVARI, 1933; GIACOBBE 1939; BIONDI, 1972, 1982; AN- } \\
\text { ZALONE \& al., 1977; BRULlo \& GUARINO 1998; BIONDI \& al., 2002; FILI- } \\
\text { BECK, 2006); Sicily (BRULlO \& MARCENÒ, 1985; GIANGUZZI \& al., 1996); } \\
\text { Sardinia (CHIAPPINI 1960, BACCHETTA \& al., 2007); Iberian peninsula (DÍAS } \\
\text { GONZÁLEZ \& FERNÁNDEZ PRIETO 1974; RIVAS-MARTÍNEZ, 1975; BUENO } \\
\text { SÁNCHEZ \& FERNÁNDEZ 1991; LOIDI \& al., 1997; RoDRÍGUEZ GUITIÁN \& } \\
\text { al., 2007); Creete (BARBERO \& QUEZEL, 1980); Greece (BARBERO \& QUE- } \\
\text { ZEL, 1978). }\end{array}$ \\
\hline
\end{tabular}

Quercus suber

Central Italy, near Fossanova Latina (LUCCHESE, 1996).

Quercus calliprinos

Palestine (ZoHARY, 1960); Greece (KNAPP, 1965).

\section{B) DECIDUOUS WOODLAND}

\section{Quercus pubescens}

Quercus robur

Quercus cerris

Quercus virgiliana

Quercus ichnusae

Quercus canariensis

Carpinus betulus

Ulmus minor

Ostrya carpinifolia and Tilia platyphyllos
Italian penisula (BIONDI 1982; UBALDI \& al., 1984; UBALDI 1988; FILIBECK, 2006); Southern France (BARBERO \& QUEZEL, 1994).

Central Italy in Tuscany, in Migliarino and S. Rossore forests (MoNTELUCCI, 1964); northwest of Iberian peninsula (Blechno spicant-Quercetum roboris subass. lauretosum nobilis; RodRíGUEZ GUITIÁN \& al., 2007).

Lazio (LUCCHESE, 1992; FILIBECK, 2006).

Central Italy (MonTELUCCI, 1946); southeastern Sicily (Lauro nobilisQuercetum virgilianae; BRULLO \& al., 2001).

Sardinia (Ornithogalo pyrenaici-Quercetum ichnusae subass. lauretosum nobilis; BACCHETTA \& al., 2007).

Nordafrica (BARBERo \& al., 1981; BARBERo \& QUEZEL, 1994).

Central Italy in Lazio coastal area, in Bracciano and Tolfa mountains (SPADA, 1977; LuCChese \& PignatTi, 1990).

Central Italy in Lazio (LePORATTI \& LATTANZI, 1996).

Sicily in Peloritani mountains (BARTOLO \& al., 1990).

\section{C) RIPARIAN WOODLANDS}

\begin{tabular}{|c|c|}
\hline Alnus glutinosa & Northern Italy (BRULLO \& GUARINO, 1998). \\
\hline Populus canescens & Central Italy in Marche and Umbria, (AlLEGREZZA \& al., 2006). \\
\hline Salix pedicellata and Populus nigra & $\begin{array}{l}\text { Northwestern Sicily (Ulmo canescentis-Salicetum pedicellatae; BRULLO } \\
\text { \& SPAMPINATO, 1990). }\end{array}$ \\
\hline Salix atrocinerea & $\begin{array}{l}\text { Sardinia (Myrto communis-Salicetum atrocinereae subass. lauretosum no- } \\
\text { bilis; BACCHETTA \& al., 2007). }\end{array}$ \\
\hline Fraxinus angustifolia subsp. oxycarpa & $\begin{array}{l}\text { Albany (KARPATI \& KARPATI , 1961); Italian peninsula (PEDROTTI \& GAFTA, } \\
\text { 1992). }\end{array}$ \\
\hline Liquidambar orientalis & Southern Anatolia (AKMAN \& al., 1978; 1993). \\
\hline
\end{tabular}


around Palermo, in Oreto valley (GianguZZI \& al., 1995); -the Hyblaean mountains, along the slopes of Mount Lauro (BRULLO \& al., 2001); in the Nature Reserve Grotta di Santa Ninfa (PASTA \& LA MANTIA, 2001); -Nebrodi, near Frazzanò (Gianguzzi, 1999; Brullo \& al., 2002); -Vallone Cerasa in Mezzojuso (BRULlo \& al., 2002); -Bosco della Ficuzza (Brullo \& SPAMPINATO 1990; GianguZZI, 2004); -Erice mountain (SCUDERI, 2006).

A census has been already taken of most of these biotopes in the context of the research project on priority habitats in Italy (Council Directive 92/43/EEC), under the coordination of the Italian Botanical Society, on behalf of the Ministry of Environment (BRULLO \& al., 2002).

\section{PHYTOSOCIOLOGY AND SYNTAXONOMY}

From the phytosociological point of view, the first described syntaxon relative to a bay laurel formation is the Hedero helicis-Lauretum nobilis, proposed by BuEno SÁNCHEZ \& FERnÁndeZ PRIETO (1991) - occurring along the Atlantic coasts of the Iberian peninsula, between Cantabria and Asturias. The association is described as pre-forest edaphophilous and calcicolous vegetation, occurring on rocky outcrops, included in the PistacioRhamnetalia alaterni order. In other studies the syntaxon is refers to Arbuto-Laurion nobilis, a new alliance, including shrubby and woody vegetation aspects abundant in laurophyllous species, with a shade-loving character, occurring in an oceanic bioclimate (RIVAS MARTíNEZ \& LOIDI, 1988; LOIDI \& al., 1997; RIVAS-MARTÍNEZ \& al., 1999).

Another laurel association - described on the basis of a single survey - was proposed by DíAZ GonzÁlez \& Fernández PRIETO (1994), as Calluno vulgaris-Lauretum nobilis (PRIETO \& al., 1994), a thermophilous and subalophilous formation, occurring along the Galician and Asturian coasts. The association was subsequently reconfirmed by Álvarez ARBESÚ (2005) and RodRíGUEZ GUITIÁN \& al. (2007) who also clarify its floristic characterization. According to these authors, the coenosis is typical in particular of a thermotemperate thermotype (with an ombrotype ranging from upper-sub-humid to lower-humid), in soils developed from in situ alteration of siliceous rocks.

On the data from the above-quoted chart presented by BuENo SÁNCHEZ \& FERNÁNDEZ PRIETO (1991), once more Díaz GonzÁLEZ \& FERnÁNDEZ PRIETO, 1994) Hedero helicis-Lauretum nobilis is differentiated into two sub-associations: the lauretosum nobilis (referring to the typical aspect, related to decidedly coastal and more or less salty environments) and the euphorbietosum amygdaloidis (more typical of inland areas).

A third association -recently proposed by HORNADO \& al. (2003)- is Omphalodo nitidaeLauretum nobilis, occurring over siliceous substrata in north-east Portugal.

More recently, RodRíguez GUITIÁN \& al. (2007), in a study on lauroid formations in the Cantabrian mountains (northeast Iberia), proposed, two further Laurel associations for this area: Tamo communis-Lauretum nobilis - calcicolous coenosis, already known sub Hedero helicis-Lauretum nobilis euphorbietosum amygdaloidis (Díaz GonzÁlez \& Fernández Prieto, 1994) and Holco mollis-Lauretum nobilis, with an acidloving character.

Other similar vegetation aspects with $L$. nobilis have been indicated for the Balearic Islands, in the Serra de Tramuntana of Majorca (Moragues \& Moragues, 2004). However, no phytosociological data or syntaxonomic reference was cited in the study. Among other species, it refers to the presence of Hedera helix, Ruscus aculeatus, Viburnum tinus and Asplenium onopteris.

In the Mediterranean area, further laurel vegetation is cited by BRULLO \& al. (2001) for south east Sicily (Hyblaean mountains), and refers to Hedero helicis-Lauretum nobilis, an association indicated also for Kefalonia Island (Greece), based on the surveys of BoLòs \& al. (1996).

Further laurel associations, occurring in oceanic climate, have recently been described in Italy and included in the alliance Fraxino orniQuercion ilicis (Quercetalia ilicis). They are: Rusco hypoglossi-Lauretum nobilis (BIONDI \& al., 2004), occurring in the central Apennines; Fraxino orni-Lauretum nobilis, indicated for the 
hilly area of the Adriatic sector (ALLEGREZZA \& al., 2006); - Celtido australis-Lauretum nobilis (BACCHETTA \& al., 2007), localized on effusive and metamorphic volcanic substrata in central and north-west Sardinia.

\section{DATA AND METHODS}

The distributional update of vegetation aspects for the habitat "Arborescent Matorral with Laurus nobilis (5230)" in Sicily, was carried out based on bibliographical data and field research (Figure 1). Table 2 shows the main characteristics of these sites, in addition to information about altitude, geolithological and bioclimatic data. Grid references used refers to the Floristic Map of Central Europe (Pignatti, 1978).

Vegetation analysis, carried out according to the classic sigmatist method of the Zurigo-Montpellier school (BRAUN-BLANQUET, 1932), was based on 18 phytosociological relevés, as well as on other avai- lable bibliographic data concerning the vegetation aspect of Mount Lauro (BRULLO \& al., 2001).

In order to make floristic-phytocoenotic comparisons, we considered further L. nobilis communities described for the Mediterranean and Atlantic areas whose data are summarized in synthetic columns of Table 4 . These refer in particular to the aspects reported in BRULLO \& al. (2001) for the Hyblaean area (SE Sicily), as well as to aspects proposed for associations already described on the Italian peninsula (ALLEGREZZA $\& a l ., 2006)$ and the Iberian peninsula (BUENO SÁnchez \& Fernández Prieto, 1991; Díaz GonzÁlez \& Fernández Prieto, 1994; HonRADO \& al., 2003; RODRíGUEZ GUITIÁN \& al., 2007).

Taxonomic nomenclature of the species in Table 3 refers to PignatTi (1982) and to the more recent updates reported in the checklist of CONTI \& al. (2005). Nomenclature of the other taxa in Table 4 follows CASTROviejo \& al., (1986-2003) and Tutin \& al., 1964-80 and 1993.

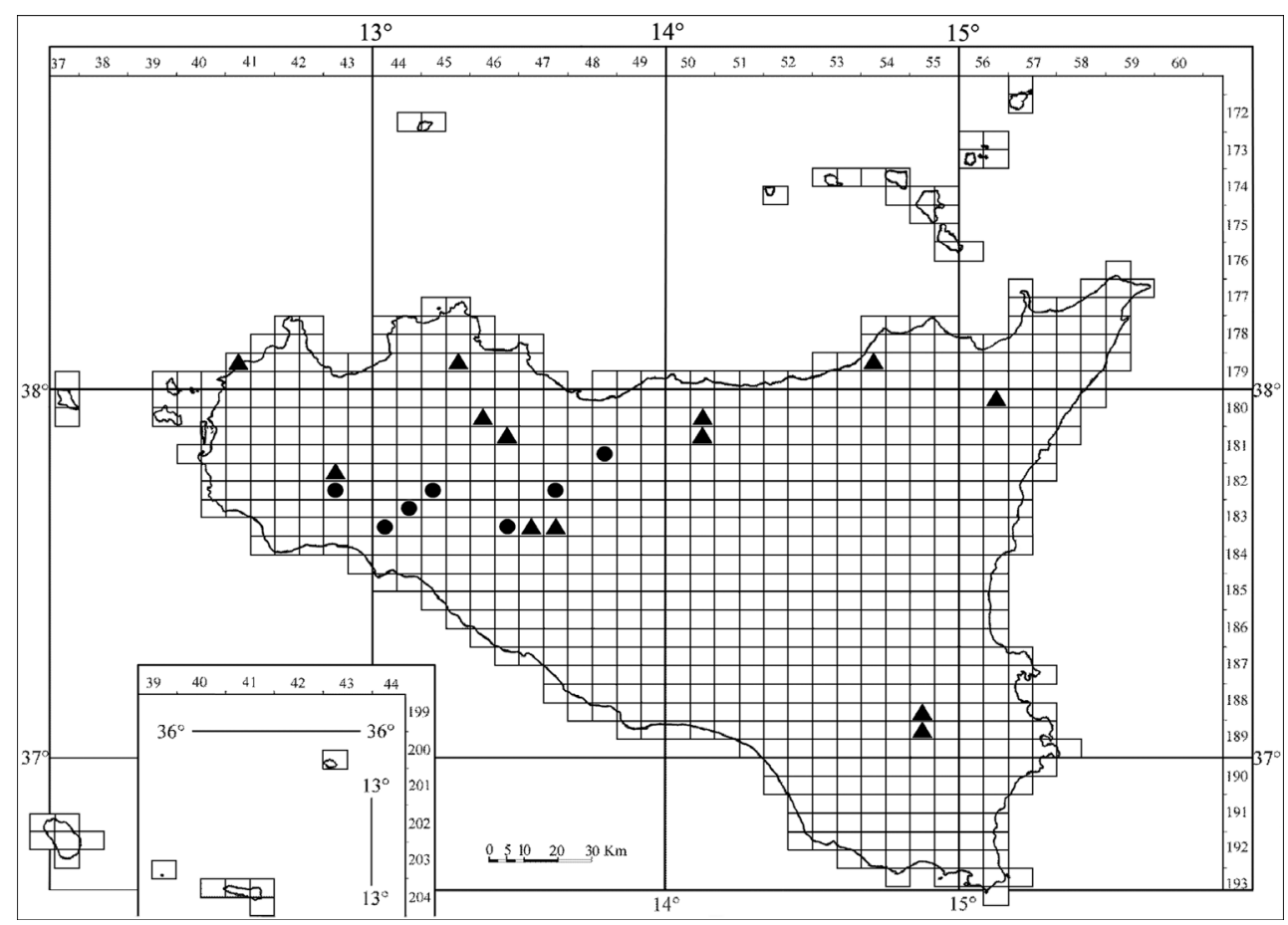

Figure 1. - Distribution of Laurus nobilis woodlands in Sicily (triangles refer to previously known sites from bibliography; circles indicate the new and unpublished sites). 
Table 2

General data of studied sites.

\begin{tabular}{|c|c|c|c|c|}
\hline $\begin{array}{l}\text { Site } \\
\text { Locality }\end{array}$ & $\begin{array}{l}\text { Mean } \\
\text { elevation } \\
\text { (m s.l.m.) }\end{array}$ & $\begin{array}{l}\text { Surface } \\
\left(\mathrm{m}^{2}\right)\end{array}$ & Lithology & $\begin{array}{c}\text { Thermotype and } \\
\text { ombrotype } \\
\text { (Mediterranean pluviseasonal } \\
\text { ocanic bioclimate) }\end{array}$ \\
\hline Santa Ninfa (TP), Contrada Biviere & 450 & 250 & Gypseous clay & $\begin{array}{l}\text { Mesomediterranean } \\
\text { lower subhumid }\end{array}$ \\
\hline $\begin{array}{l}\text { Partanna (TP), Vallone Binaia } \\
\text { in Contrada Stretto }\end{array}$ & 280 & 1.500 & Gypseous clay & $\begin{array}{l}\text { Thermomediterranean } \\
\text { lower subhumid }\end{array}$ \\
\hline $\begin{array}{l}\text { Sambuca di Sicilia (AG), } \\
\text { Contrada Arancio }\end{array}$ & 300 & 6.000 & Limestones & $\begin{array}{c}\text { Thermomediterranean } \\
\text { upper dry }\end{array}$ \\
\hline $\begin{array}{l}\text { Sambuca di Sicilia (AG), } \\
\text { Contrada Menta }\end{array}$ & 80 & 8.000 & $\begin{array}{c}\text { Clays and } \\
\text { limestone marls }\end{array}$ & $\begin{array}{l}\text { Thermomediterranean } \\
\text { lower subhumid }\end{array}$ \\
\hline Bivona (AG), Torrente Alba & 615 & 1.000 & Limestone marls & $\begin{array}{l}\text { Mesomediterranean } \\
\text { lower subhumid }\end{array}$ \\
\hline Menfi (AG), Monte Arancio & 330 & 3.000 & $\begin{array}{l}\text { Calcarenites } \\
\text { and sands }\end{array}$ & $\begin{array}{c}\text { Thermomediterranean } \\
\text { upper dry }\end{array}$ \\
\hline Sciacca (AG), Lago Arancio & 240 & 2.000 & $\begin{array}{l}\text { Calcarenites } \\
\text { and sands }\end{array}$ & $\begin{array}{c}\text { Thermomediterranean } \\
\text { upper dry }\end{array}$ \\
\hline Bisacquino (PA), Contrada Alvano & 375 & 400 & $\begin{array}{l}\text { Marls and } \\
\text { sandy clays }\end{array}$ & $\begin{array}{l}\text { Thermomediterranean } \\
\text { lower subhumid }\end{array}$ \\
\hline Bisacquino (PA), Contrada Gallinaro & 475 & 300 & Sandy clays & $\begin{array}{l}\text { Thermomediterranean } \\
\text { lower subhumid }\end{array}$ \\
\hline Castronovo di Sicilia (PA), Ponte Morello & 450 & 300 & $\begin{array}{l}\text { Clayey marls } \\
\text { and alluvium }\end{array}$ & $\begin{array}{l}\text { Mesomediterranean } \\
\text { upper dry }\end{array}$ \\
\hline $\begin{array}{l}\text { Montemaggiore Belsito (PA), } \\
\text { Bosco della Favara }\end{array}$ & 800 & 300 & Sandstone & $\begin{array}{l}\text { Mesomediterranean } \\
\text { upper dry }\end{array}$ \\
\hline Buccheri e Buscemi (SR), Monte Lauro & 700 & 500 & Limestone marls & $\begin{array}{l}\text { Mesomediterranean } \\
\text { upper dry }\end{array}$ \\
\hline
\end{tabular}

\section{RESULTS AND DISCUSSION}

From the elaboration of $L$. nobilis relevés of the vegetation -carried out in different Sicilian sites (Table 3)- and from comparison with the synthetic columns referring to the other L. nobilis coenosis already noted (Table 4), it can be seen that there is a clear syntaxonomic independence of the formations in the study. Based on the floristic, phytogeographical and ecological peculiarities of the Sicilian aspects we propose that they should be attributed to a new association Acantho mollis-Lauretum nobilis ass. nova hoc loco (Table 3 , holotypus rel. 14), that we consider as vicariant for the south-central Mediterranean area.

The association is dominated by L. nobilis, and shows a relevant abundance of some other laurophyllous species (Hedera helix, Rhamnus alaternus subsp. alaternus, Ruscus aculeatus, Smilax aspera and sometimes also Viburnum tinus), lianas (Rubia peregrina var. longifolia, Asparagus acutifolius, Tamus communis, Clematis vitalba, Calystegia sylvatica and Rubus ulmifolius) together with few other broad-leaved grasses in the undergrowth such as Acanthus mollis and Cyclamen hederifolium subsp. confusum. The last two entities are both included in the characteristic species combination, together with Pistacia terebinthus and Orobanche hederae.

Data in Table 3 reveal an extreme floristic poverty of L. nobilis woodland, since their tree layer markedly reduces light intensity at the soil level. In the undergrowth, grasses and ferns are rather reduced, 
Table 3

Acantho mollis-Lauretum nobilis ass. nova

(Arbuto unedonis-Laurion nobilis, Pistacio lentisci-Rhamnetalia alaterni, Quercetea ilicis)

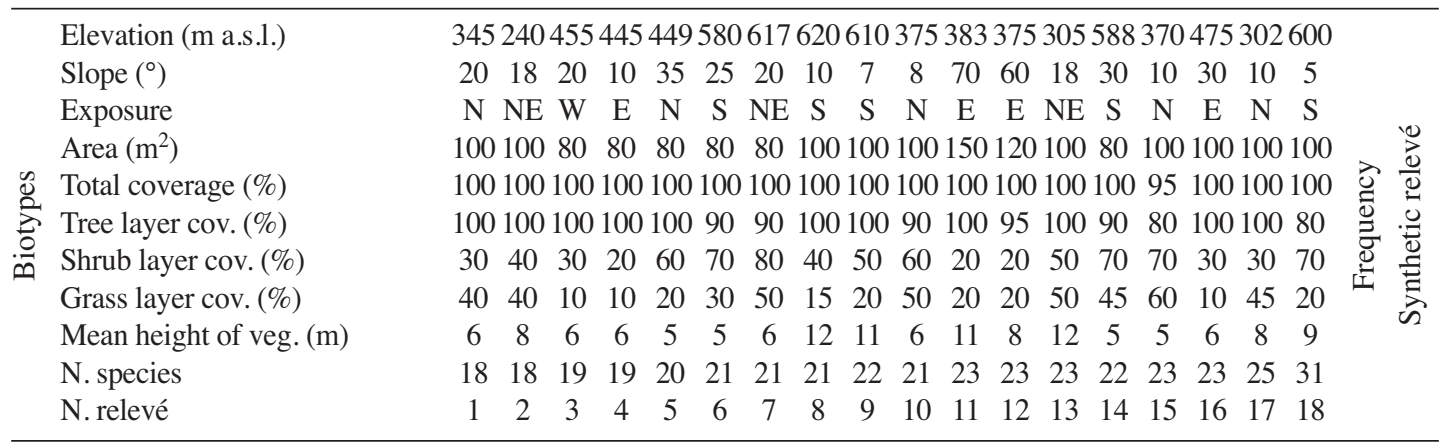

Characteristics of assoc.

$\mathrm{P}$ Laurus nobilis

$\mathrm{P}$ Hedera helix

$\begin{array}{llllllllllllllllllll}5 & 5 & 5 & 5 & 5 & 5 & 5 & 5 & 5 & 5 & 5 & 5 & 5 & 5 & 5 & 5 & 5 & 5 & 18 & \mathrm{~V}\end{array}$

Characteristics and

differentials of assoc.

$\mathrm{H}$ Acanthus mollis

$\mathrm{G}$ Cyclamen hederifolium subsp. confusum

P Pistacia terebinthus

$\mathrm{T}$ Orobanche hederae

Characteristics of Pistacio-

Rhamnetalia and Quercetea ilicis

$\begin{array}{lllllllllllllllllllll}\text { NP Asparagus acutifolius } & 1 & 1 & 1 & 2 & 2 & 2 & 1 & + & 1 & 2 & + & 1 & 1 & 2 & 1 & 2 & 1 & 1 & 18 & \mathrm{~V}\end{array}$

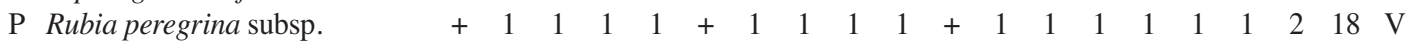
longifolia

G Arisarum vulgare

$\mathrm{G}$ Allium subhirsutum

NP Smilax aspera

G Tamus communis

NP Euphorbia characias

P Rhamnus alaternus

NP Osyris alba

NP Rosa sempervirens

$\mathrm{P}$ Fraxinus ornus

P Quercus virgiliana

H Carex distachya

$\mathrm{P}$ Celtis australis

$\mathrm{P}$ Chamaerops humilis

$\mathrm{G}$ Ruscus aculeatus

$\mathrm{H}$ Asplenium onopteris

P Quercus ilex

P Viburnum tinus

P Rhamnus oleoides

$\begin{array}{llllllllllllllllllll}2 & 2 & 3 & 2 & 2 & 1 & + & 3 & 2 & 3 & 3 & 2 & 2 & 2 & 3 & 3 & 1 & 2 & 18 & \mathrm{~V}\end{array}$

$211+++.111111+1++2217 \mathrm{~V}$

$\begin{array}{cccccccccccccccccccccc}2 & 1 & \cdot & 1 & 1 & 1 & 2 & \cdot & 1 & \cdot & 1 & . & 1 & 2 & \cdot & 1 & \cdot & \cdot & 10 & \text { III } \\ + & . & . & + & . & + & + & . & . & . & + & + & . & + & . & . & . & . & 6 & \text { II }\end{array}$

Trasgressives of Salici-Populetea

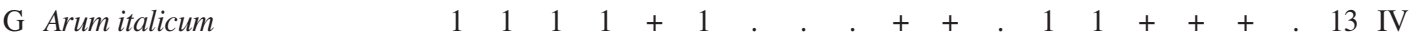

$\begin{array}{lllllllllllllllllllllllllll}\mathrm{P} & \text { Ficus carica } \text { var. caprificus } & 1 & 1 & . & . & 1 & . & . & 1 & . & . & 1 & 1 & 1 & . & . & . & 1 & 1 & 9 & \text { III }\end{array}$

P Populus nigra

$\mathrm{H}$ Brachypodium sylvaticum

$\mathrm{P}$ Ulmus minor

H Carex pendula

\begin{tabular}{|c|c|c|c|c|c|c|c|c|c|c|c|c|c|c|c|c|c|c|c|}
\hline & + & + & 1 & & & & $t$ & 1 & & & 1 & & & & & & + & 18 & V \\
\hline ] & + & 1 & 1 & + & + & + & 1 & 2 & + & & + & 1 & + & 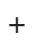 & + & + & 1 & 17 & V \\
\hline & 2 & 3 & 2 & 1 & + & & 1 & + & 2 & ? & 2 & 1 & & 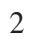 & 3 & & 1 & 14 & IV \\
\hline & 1 & 1 & 1 & 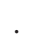 & . & + & 1 & 1 & 2 & & + & + & + & 1 & + & + & 1 & 14 & \\
\hline & . & $\cdot$ & . & . & + & & . & + & + & & & + & + & $t$ & + & 1 & + & 9 & \\
\hline & 1 & 1 & 2 & 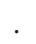 & + & 2 & 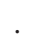 & . & & 1 & 1 & & 3 & & . & 1 & & 9 & \\
\hline & 1 & $\cdot$ & . & . & + & + & 2 & 2 & 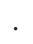 & & . & & + & & 1 & & + & 8 & \\
\hline & . & . & . & . & . & . & 1 & 1 & . & + & + & $\cdot$ & 1 & . & 2 & & 1 & 7 & \\
\hline & . & 1 & . & 2 & 1 & . & $\cdot$ & . & 1 & 1 & . & . & . & $\cdot$ & . & & 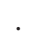 & 5 & \\
\hline & . & . & 1 & r. & . & . & . & 1 & . & . & $v^{\prime}$ & . & . & . & + & . & 1 & 4 & II \\
\hline & . & . & . & $\cdot$ & . & . & 1 & + & . & + & . & . & . & 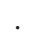 & . & . & + & 4 & II \\
\hline & . & . & . & . & 1 & . & . & . & . & 1 & 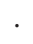 & . & 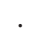 & 1 & . & $\cdot$ & $\cdot$ & 3 & \\
\hline & . & . & . & $\cdot$ & . & . & . & . & . & . & $\cdot$ & + & . & . & . & 1 & . & 2 & I \\
\hline & . & . & . & . & . & . & . & . & . & . & . & . & + & $\cdot$ & 1 & . & . & 2 & I \\
\hline & . & 1 & . & $\cdot$ & . & . & . & . & . & . & $\cdot$ & . & . & . & . & . & . & 1 & \\
\hline & . & . & . & $\cdot$ & . & . & . & . & . & . & . & . & . & 1 & . & 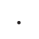 & . & 1 & \\
\hline & . & . & . & . & . & . & . & . & . & . & . & . & . & . & 1 & . & . & 1 & \\
\hline & . & . & . & . & . & . & . & . & 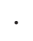 & . & . & . & . & . & . & 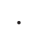 & . & 1 & I \\
\hline 1 & 1 & 1 & 1 & + & 1 & . & & . & + & + & . & 1 & 1 & + & + & + & . & 13 & \\
\hline 1 & 1 & . & . & 1 & $\cdot$ & . & 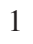 & · & $\cdot$ & 1 & 1 & 1 & . & $\cdot$ & $\cdot$ & 1 & 1 & 9 & \\
\hline . & . & . & . & $\cdot$ & . & . & 1 & . & . & 2 & 2 & . & . & · & . & 1 & 1 & 5 & \\
\hline+ & . & . & . & . & . & + & + & . & . & . & . & . & . & . & . & + & + & 5 & \\
\hline & 1 & . & . & $\cdot$ & . & . & . & 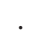 & . & & 2 & . & . & . & . & . & + & 4 & \\
\hline & . & . & . & . & 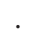 & . & . & 1 & . & & + & 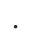 & 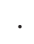 & · & . & . & 1 & 3 & \\
\hline
\end{tabular}




\begin{tabular}{|c|c|c|c|c|c|c|c|c|c|c|c|c|c|c|c|c|c|c|c|c|}
\hline & N. relevé & 1 & 2 & 3 & 4 & 5 & 6 & 7 & 8 & 9 & 10 & 11 & 12 & 13 & 14 & 15 & 16 & 17 & 18 & \\
\hline $\mathrm{P}$ & Populus alba & . & $r_{0}$ & . & . & . & . & . & . & . & . & $\cdot$ & $\cdot$ & 3 & . & . & 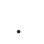 & 2 & 2 & I \\
\hline $\mathrm{P}$ & Salix pedicellata & . & . & 1 & . & 1 & . & $\cdot$ & . & . & . & $\cdot$ & 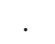 & . & . & $\cdot$ & . & $\cdot$ & 2 & I \\
\hline $\mathrm{P}$ & Tamarix africana & . & . & . & . & $\cdot$ & . & . & . & · & . & . & . & . & . & 1 & . & . & 1 & I \\
\hline $\mathrm{P}$ & Sambucus nigra & . & . & . & . & . & . & $\cdot$ & . & . & . & . & . & . & . & . & . & 1 & 1 & I \\
\hline NP & Solanum dulcamara & . & . & . & . & . & . & . & . & . & . & . & + & . & . & . & . & . & . 1 & I \\
\hline $\mathrm{G}$ & Equisetum telmateja & . & . & . & . & . & . & $\cdot$ & . & . & . & . & + & . & . & . & . & . & . 1 & I \\
\hline NP & Hypericum hircinum & . & . & . & . & . & . & . & . & . & . & . & . & . & . & . & . & . & +1 & I \\
\hline & Trasgressives of Rhamno-Prl & & & & & & & & & & & & & & & & & & & \\
\hline NP & Rubus gr. ulmifolius & 2 & 1 & 1 & 2 & 3 & 2 & 2 & 1 & 2 & 2 & + & 1 & 1 & 2 & 2 & 1 & 2 & 218 & V \\
\hline $\mathrm{P}$ & Clematis vitalba & . & . & 2 & 2 & 1 & + & . & 1 & 1 & 2 & 1 & . & . & 1 & 1 & 1 & . & 112 & IV \\
\hline $\mathrm{H}$ & Calystegia sylvatica & . & 1 & . & . & . & + & . & 1 & + & 1 & . & . & + & . & + & + & + & 110 & III \\
\hline $\mathrm{P}$ & Prunus spinosa & . & . & . & . & . & . & + & . & . & 1 & . & . & . & 1 & 2 & . & + & . 5 & II \\
\hline $\mathrm{P}$ & Crataegus monogyna & . & . & . & . & . & . & . & . & 1 & . & . & . & 1 & . & . & . & 1 & +4 & II \\
\hline NP & Rhus coriaria & . & . & . & . & 1 & . & 1 & . & . & . & . & . & . & . & . & . & . & . 2 & I \\
\hline $\mathrm{P}$ & Pyrus spinosa & . & . & . & . & . & . & . & . & . & . & . & . & . & . & 1 & 1 & . & . 2 & I \\
\hline NP & $\begin{array}{l}\text { Rosa canina } \\
\text { Companions }\end{array}$ & . & . & . & . & 1 & . & . & . & . & . & . & . & . & . & . & . & . & . 1 & I \\
\hline $\mathrm{H}$ & Smyrnium olusatrum & 1 & + & . & . & $\cdot$ & + & + & 1 & . & 1 & . & . & 1 & + & 1 & . & + & +11 & IV \\
\hline $\mathrm{H}$ & Piptatherum miliaceum & + & & . & . & . & . & 1 & . & 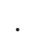 & . & . & . & . & 1 & + & + & + & . 6 & II \\
\hline G & Arundo collina & . & . & 1 & 1 & 1 & . & $\cdot$ & . & . & . & + & . & . & . & . & . & . & . & II \\
\hline $\mathrm{H}$ & Ampelodesmos mauritanicus & . & . & . & . & . & . & . & . & . & 1 & + & . & . & . & 1 & . & 1 & . & II \\
\hline $\mathrm{G}$ & Oxalis pes-caprae & . & . & + & + & + & . & $\cdot$ & $\cdot$ & . & $\cdot$ & . & . & $\cdot$ & . & . & 1 & . & . & II \\
\hline $\mathrm{H}$ & Parietaria judaica & . & . & . & . & . & . & + & + & . & . & . & . & + & . & . & . & . & +4 & II \\
\hline $\mathrm{G}$ & Allium triquetrum & . & . & . & . & . & . & $\cdot$ & . & . & + & + & & . & . & + & . & . & . 3 & I \\
\hline G & Adiantum capillus-veneris & . & . & . & . & $\cdot$ & . & $\cdot$ & . & . & . & 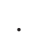 & 1 & . & . & . & . & . & $\mathrm{r} \quad 2$ & I \\
\hline $\mathrm{H}$ & Carex divulsa & . & . & . & . & . & . & $\cdot$ & . & . & + & $\cdot$ & . & . & . & + & . & . & . & I \\
\hline & $\begin{array}{l}\text { Polypodium cambricum } \\
\text { subsp. serrulatum }\end{array}$ & . & . & . & . & & . & $\cdot$ & . & · & . & + & + & . & . & . & . & . & . & I \\
\hline
\end{tabular}

Companions: Cirsium scabrum 1 in 1; Thalictrum calabricum + in 4; Euphorbia ceratocarpa + in 5; Biarum tenuifolium 1 in 7; Ballota rupestris + in 9; Arundo donax 1 in 13; Dorycnium rectum + in 13; Ferula communis + in 14; Geranium robertianum, Picris echioides and Polypogon viridis + in 18.

Localities:1: Menfi, Agrigento, Arancio Mountain, 14.11.2007; 2: Sciacca, Agrigento, eastern from the weir of the Lake Arancio, 14.11 .2007; 3-4: Castronovo di Sicilia, Palermo, Ponte Morello, 8.05.2007; 5 : Nature Reserve S. Ninfa, Trapani, Contrada Biviere, 14.05.2007; 6-7: Sambuca di Sicilia, Palermo, Contrada Menta, 5.12.2006; 8-9: Bivona, Agrigento, Torrente Alba, 24.05.2007; 10: Sambuca di Sicilia, Palermo, Contrada Menta, 5.12.2006; 11-12: Partanna, Trapani, Vallone Binaia, in Contrada Stretto, 20.03.2008; 13: Sambuca di Sicilia, Agrigento, Contrada Arancio, 14.11.2007; 14: Sambuca di Sicilia, Palermo, Contrada Menta, 5.12.2006 holotypus ass.; 15: Bisacquino, Agrigento, Contrada Alvano, 4.05.2007; 16: Bisacquino, Agrigento, Contrada Gallinaro, 4.05.2007; 17: Sambuca di Sicilia, Agrigento, Contrada Arancio, 14.11.2007; 18: Bivona, Agrigento, Torrente Alba, 24.05.2007.

as compared to a notable abundance of bryophytes and lichens. A considerable presence of shaded leaves can be observed in the same L. nobilis individuals - together with dried leaves on lower branches, showing cortical necrosis caused by fungi.

Regarding the phytosociological floristic aspect, there is an abundance of elements from Quercetea ilicis (the following also appear, in addition to the already-listed species: Euphorbia characias, Osyris alba, Carex distachya, etc.), Salici purpureae-Populetea nigrae (Arum itali- cum, Ficus carica var. caprificus, Populus nigra, Populus alba, Salix pedicellata, Sambucus nigra, etc.) and Rhamno-Prunetea (Rubus ulmifolius, Clematis vitalba, Prunus spinosa, Crataegus monogyna, etc.).

Following the syntaxonomic classification proposed by other authors (BRULlo \& al., 2001; RODRÍGUEZ GUITIÁN \& al., 2007), the coenosis was referred to the Arbuto unedonis-Laurion nobilis alliance and to the order Pistacio lentisciRhamnetalia alaterni. 
Table 4

Simplified synoptic table of Laurus nobilis communities in Mediterranean and Atlantic area

\begin{tabular}{|c|c|c|c|c|c|c|c|c|c|c|c|c|c|c|}
\hline Number of relevés & 18 & 10 & 15 & 4 & 7 & 1 & 1 & 41 & 27 & 4 & 19 & 21 & 9 & 9 \\
\hline Number of table & 1 & 2 & 3 & 4 & 5 & 6 & 7 & 8 & 9 & 10 & 11 & 12 & 13 & 14 \\
\hline Characteristics and differentials of assoc. & & & & & & & & & & & & & & \\
\hline & V & V & V & 4 & V & 5 & 4 & $\mathrm{~V}$ & $\mathrm{~V}$ & 4 & V & V & V & $\mathrm{V}$ \\
\hline Hedera helix & $\mathrm{V}$ & $\mathrm{V}$ & $\mathrm{V}$ & 4 & V & 2 & 3 & $\mathrm{~V}$ & V & 4 & $\mathrm{~V}$ & V & . & $\mathrm{V}$ \\
\hline Acanthus mollis & $\mathrm{V}$ & $\mathrm{V}$ & I & . & . & . & . & . & . & . & . & . & . & . \\
\hline Cyclamen hederifolium subsp. confusum & $\mathrm{V}$ & $\mathrm{V}$ & . & . & . & . & . & . & . & . & . & . & . & . \\
\hline Pistacia terebinthus & III & II & . & . & . & . & . & . & . & . & . & . & . & . \\
\hline Orobanche hederae & II & I & I & . & . & . & . & . & . & . & . & . & . & . \\
\hline Celtis australis & I & III & III & . & . & . & . & . & . & . & . & . & . & . \\
\hline Ficus carica var. caprificus & II & II & III & . & . & . & . & . & . & . & . & . & . & $\cdot$ \\
\hline Cyclamen repandum & . & . & II & . & . & . & . & . & . & . & . & . & . & . \\
\hline Fraxinus ornus & I & . & . & 3 & . & . & . & . & . & . & . & . & . & . \\
\hline Cyclamen hederifolium & . & . & . & 1 & & . & . & . & . & . & . & . & . & . \\
\hline Daucus gummifer & . & . & . & . & II & . & . & . & . & . & . & . & . & . \\
\hline Asparagus prostratus & . & . & . & . & $\mathrm{I}$ & . & . & . & . & . & . & . & . & . \\
\hline Rumex biformis & . & . & . & . & II & . & . & . & . & . & . & . & . & . \\
\hline Leucanthemum crassifolium & . & . & . & . & II & . & . & . & . & . & . & . & . & . \\
\hline Genista occidentalis & . & . & . & . & IV & . & . & . & . & . & . & . & . & . \\
\hline Lithodora diffusa & . & . & . & . & IV & . & . & . & . & . & . & . & . & . \\
\hline Silene divaricata & . & . & . & . & III & . & . & . & . & . & . & . & . & . \\
\hline Vincetoxicum hirundinaria & . & . & . & . & II & . & . & . & . & . & . & . & . & . \\
\hline Carduus argemone & . & . & . & . & II & . & . & . & . & . & . & . & . & . \\
\hline Scabiosa columbaria & . & . & . & . & I & . & . & . & . & . & . & . & . & . \\
\hline Crepis albida & . & . & . & . & I & . & . & . & . & . & . & . & . & . \\
\hline Dianthus monspessulanum & . & . & . & . & $\mathrm{I}$ & . & . & . & . & . & . & . & . & . \\
\hline Rumex acetosa subsp. biformis & . & . & . & . & . & . & + & I & II & . & . & . & . & . \\
\hline Silene uniflora & . & . & . & . & . & . & + & I & II & . & . & . & . & . \\
\hline Leucanthemum pluriflorum & . & . & . & . & . & . & + & I & II & . & . & . & . & . \\
\hline Crithmum maritimum & . & . & . & . & I & . & . & . & III & . & . & . & . & . \\
\hline Festuca rubra subsp. pruinosa & . & . & . & . & . & . & 1 & . & III & . & . & . & . & . \\
\hline Calluna vulgaris & . & . & . & . & . & . & 3 & . & . & . & . & . & . & . \\
\hline Daucus carota subsp. gummifer & . & . & . & . & . & . & . & . & I & . & . & . & . & $\cdot$ \\
\hline Inula crithmoides & . & . & . & . & . & . & . & . & I & . & . & . & . & . \\
\hline Brassica oleracea var. sylvestris & . & . & . & . & . & . & . & . & I & . & . & . & . & . \\
\hline Armeria maritima & . & . & . & . & . & . & . & . & I & . & . & . & . & . \\
\hline Asplenium marinum & . & . & . & . & . & . & . & . & $\mathrm{I}$ & . & . & . & . & . \\
\hline Mercurialis perennis & . & . & . & . & II & . & . & I & I & 2 & II & $\mathrm{I}$ & . & II \\
\hline Iris foetidissima & . & . & . & . & II & . & . & . & III & 1 & II & III & . & . \\
\hline Cornus sanguinea & . & . & . & 2 & III & + & . & . & . & 3 & . & II & . & . \\
\hline Phyllitis scolopendrium & . & . & . & . & & . & . & I & II & . & II & III & . & I \\
\hline Primula acaulis & . & . & . & . & . & . & . & I & II & . & . & I & . & $\mathrm{I}$ \\
\hline Hypericum androsaeum & . & . & . & . & . & . & - & . & I & . & $\mathrm{I}$ & I & I & . \\
\hline Ilex aquifolium & . & . & . & . & . & . & . & . & I & . & . & II & . & I \\
\hline Fraxinus excelsior & . & . & . & . & . & + & . & . & . & . & . & III & 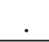 & 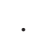 \\
\hline Omphalodes nitida & . & . & . & . & . & . & + & I & I & . & . & . & III & II \\
\hline Athyrium filix foemina & . & . & . & . & . & . & . & I & I & . & . & I & IV & I \\
\hline Salix atrocinerea & . & . & . & . & . & . & . & I & . & . & . & . & III & I \\
\hline Osmunda regalis & . & . & . & . & . & . & - & . & I & . & . & . & IV & . \\
\hline Acer pseudoplatanus & . & . & . & . & . & . & . & . & . & . & . & I & II & . \\
\hline Blechnum spicant & . & . & . & . & . & . & . & . & . & . & . & . & III & I \\
\hline Woodwardia radicans & . & . & . & . & . & . & . & . & . & . & . & . & III & . \\
\hline Frangula alnus & . & . & . & . & . & . & . & . & . & . & . & . & II & . \\
\hline Fraxinus angustifolia & . & . & . & . & . & . & $\cdot$ & . & . & . & . & . & II & . \\
\hline Hedera hibernica & . & . & . & . & . & . & . & . & . & . & . & . & $\mathrm{V}$ & \\
\hline
\end{tabular}


Number of table $\begin{array}{llllllllllllll}1 & 2 & 3 & 4 & 5 & 6 & 7 & 8 & 9 & 10 & 11 & 12 & 13 & 14\end{array}$

Saxifraga spathularis

Crepis lampsanoides

Dryopteris affinis

Holcus mollis

Oxalis acetosella

Rumex acetosa

Dryopteris dilatata

Characteristics of Pistacio-Rhamnetalia and. Quercetea ilicis

Rubia peregrina s.l.

Smilax aspera

Ruscus aculeatus

Tamus communis

Asplenium onopteris

Rosa sempervirens

Rhamnus alaternus

Osyris alba

Asparagus acutifolius

Erica arborea

Arisarum vulgare

Arbutus unedo

Carex distachya

Anagyris foetida

Clematis cirrhosa

Quercus ilex

Phillyrea latifolia

Euphorbia characias

Quercus virgiliana

Viburnum tinus

Viola alba subsp. dehnhardtii

Asparagus albus

Cyclamen hederifolium

Quercus suber

Daphne gnidium

Trasgressives of Salici-Populetea

Arum italicum

Sambucus nigra

Brachypodium sylvaticum

Ulmus minor

Solanum dulcamara

Salix pedicellata

Hypericum hircinum

Populus nigra

Vitis vinifera subsp. sylvestris

Salix alba

Populus alba

Salix purpurea

Trasgressives of Rhamno-Prunetea

Rubus gr. ulmifolius (incl. Rubus sp., col. 6-8)

Crataegus monogyna

Prunus spinosa

Lonicera periclymenum

Clematis vitalba

Companions

Polypodium cambricum

Geranium robertianum

Brachypodium rupestre

Parietaria judaica

\begin{tabular}{|c|c|c|c|c|c|c|c|c|c|c|c|c|c|}
\hline $\mathrm{V}$ & IV & II & 4 & $\mathrm{~V}$ & + & 1 & IV & V & 4 & IV & IV & II & II \\
\hline IV & III & IV & 3 & V & 2 & 2 & IV & III & 4 & $\mathrm{~V}$ & $\mathrm{~V}$ & & I \\
\hline I & $\mathrm{V}$ & IV & . & IV & 1 & + & III & IV & 4 & IV & IV & $\mathrm{V}$ & III \\
\hline IV & I & $\mathrm{V}$ & . & IV & + & . & III & IV & 4 & II & $\mathrm{V}$ & II & IV \\
\hline . & I & IV & . & II & & . & $\mathrm{V}$ & IV & . & III & III & V & III \\
\hline II & IV & II & 1 & I & + & - & . & I & 1 & . & I & . & . \\
\hline III & V & II & . & III & + & 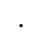 & . & I & 3 & III & III & . & . \\
\hline III & III & $\cdot$ & . & II & . & . & . & . & . & I & I & I & . \\
\hline V & III & IV & 4 & . & . & . & . & . & . & . & . & . & . \\
\hline . & . & . & . & . & . & . & I & I & . & . & . & II & I \\
\hline I & . & III & . & . & . & . & . & . & . & . & I & . & . \\
\hline W & . & $\dot{x}$ & . & . & . & . & . & I & . & . & . & III & I \\
\hline III & . & I & . & . & . & . & . & . & . & . & . & . & . \\
\hline I & I & . & . & . & . & . & . & . & . & . & . & . & . \\
\hline . & II & I & . & . & . & . & . & . & . & . & . & . & . \\
\hline . & I & . & . & . & . & . & . & . & . & I & . & . & . \\
\hline 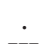 & . & III & . & . & . & . & . & . & . & . & . & . & I \\
\hline III & . & . & . & . & . & . & . & . & . & . & . & . & . \\
\hline I & . & . & . & . & . & . & . & . & . & . & . & . & . \\
\hline I & . & . & . & . & . & . & . & . & . & . & . & . & . \\
\hline . & I & . & . & . & . & . & . & . & . & . & . & . & . \\
\hline . & . & . & 3 & . & . & . & . & . & . & . & . & . & . \\
\hline . & . & . & 1 & . & . & . & . & . & . & . & . & . & . \\
\hline . & . & . & . & . & . & . & . & I & . & . & . & . & . \\
\hline$\cdot$ & . & . & . & . & . & . & . & I & . & . & . & . & . \\
\hline IV & IV & II & 1 & II & + & . & I & II & 1 & III & III & . & $\cdot$ \\
\hline I & I & I & 4 & . & . & . & II & I & . & I & II & . & I \\
\hline II & IV & . & . & . & . & . & . & I & . & . & . & II & III \\
\hline II & III & . & 1 & . & . & . & . & . & . & . & . & . & . \\
\hline I & . & . & . & I & . & . & . & . & I & . & . & . & . \\
\hline II & I & . & . & . & . & . & . & . & . & . & . & . & . \\
\hline . & I & I & . & . & . & . & . & . & . & . & . & . & . \\
\hline II & . & . & . & . & . & . & . & . & . & . & . & . & . \\
\hline . & . & II & . & . & . & . & . & . & . & . & . & . & . \\
\hline . & . & I & . & . & . & . & . & . & . & . & . & . & . \\
\hline . & . & I & . & . & . & . & . & . & . & . & . & . & . \\
\hline . & . & . & 1 & . & . & . & . & . & . & . & . & . & . \\
\hline III & V & IV & 4 & V & + & + & $\mathrm{V}$ & IV & 4 & IV & $\mathrm{V}$ & IV & $\mathrm{V}$ \\
\hline II & I & I & 1 & . & + & . & I & III & . & . & II & . & III \\
\hline III & I & I & . & III & . & . & I & II & 2 & . & III & . & I \\
\hline$\cdot$ & . & - & . & II & + & 1 & . & . & 1 & II & II & III & IV \\
\hline IV & II & II & 2 & . & . & . & . & . & . & . & I & . & I \\
\hline I & . & II & . & IV & . & + & . & I & 3 & . & II & I & I \\
\hline I & . & . & . & III & . & . & I & II & 3 & II & I & . & III \\
\hline . & . & . & . & $\mathrm{V}$ & . & . & I & $\mathrm{V}$ & 4 & IV & III & . & II \\
\hline II & II & . & . & III & . & . & I & IV & II & . & . & . &. \\
\hline
\end{tabular}




\begin{tabular}{|c|c|c|c|c|c|c|c|c|c|c|c|c|c|c|}
\hline Number of table & 1 & 2 & 3 & 4 & 5 & 6 & 7 & 8 & 9 & 10 & 11 & 12 & 13 & 14 \\
\hline Umbilicus rupestris & I & . & I & . & . & . & . & I & III & . & . & II & • & IV \\
\hline Asplenium trichomanes & . & . & II & . & I & . & . & . & . & 1 & I & II & . & III \\
\hline Teucrium scorodonia & . & . & $\mathrm{I}$ & . & . & . & 1 & III & III & . & . & I & . & IV \\
\hline Polystichum setiferum & . & . & I & . & . & . & . & III & III & . & I & II & . & III \\
\hline Quercus robur & . & . & . & . & . & $\dot{5}$ & . & I & II & . & . & II & IV & III \\
\hline Pteridium aquilinum & . & . & . & . & . & . & 1 & III & III & . & II & II & . & III \\
\hline Corylus avellana & . & . & . & . & . & . & . & . & I & . & $\mathrm{I}$ & I & II & II \\
\hline Viola riviniana & . & . & . & . & . & . & . & . & I & . & $\mathrm{I}$ & II & II & III \\
\hline Castanea sativa & . & . & . & . & . & + & . & . & $\mathrm{I}$ & . & . & II & . & II \\
\hline Asplenium adiantum-nigrum & . & . & . & . & . & . & 1 & . & III & . & $\mathrm{I}$ & II & . & . \\
\hline Ulex europaeus & . & . & . & . & . & . & 1 & I & II & . & . & I & . & \\
\hline
\end{tabular}

Procedence of relevés: 1: Acantho mollis-Lauretum nobilis ass. nov. (Table 1); 2: Acantho mollis-Lauretum nobilis ass. nov. (BRULlo \& al., 2001; Table 1); 3: Celtido australis-Lauretum nobilis (BACCHETTA \& al., 2007; Table 1, rels. 1-15); 4: Fraxino orni-Lauretum nobilis (AlLEGREZZA \& al., 1991; Table 4); 5 : Hedero helicis-Lauretum nobilis (Bueno SÁnchez \& Fernández PrIETO, 1991; Table 3); 6: Hedero helicis-Lauretum nobilis (LoIDI et al. 1997; Table 25, rel. 32); 7: Calluno vulgaris-Lauretum nobilis (DíAs GonZÁlez \& FERNÁNDEZ PrIETO, 1994; rel. type); 8 : Calluno vulgaris-Lauretum nobilis (ÁlvAREZ ARBESU, 2005); 9: Calluno vulgaris-Lauretum nobilis (RoDRíGUEZ GUITIÁN \& al., 2007; Table 3, rels. 1-27); 10: Tamo communis-Lauretum nobilis (BUENo SÁNCHEZ \& FERNÁNDEZ PRIETO, 1991; sub Hedero helicis-Lauretum nobilis subass. euphorbietoum amygdaloidis); 11: Tamo communis-Lauretum nobilis (ÁLVAREZ ARBESU, 2005; sub Hedero helicis-Lauretum nobilis); 12: Tamo communis-Lauretum nobilis (RoDRÍGUEZ GUITIÁN \& al., 2007; Table 5, rels. 1-19); 13: Omphalodo nitidae-Lauretum nobilis (HonRADO \& al., 2003); 14: Holco mollis-Lauretum nobilis (RodRíGUEZ GUITIÁN \& al., 2007; Table 5, rels. 22-30).

\section{SYNECOLOGY}

Sicily is placed in the Mediterranean Region, Western Mediterranean sub-region, Italo-Thyrrenian Province and in the Siculo sector: its area is characterized by a Mediterranean pluviseasonal oceanic bioclimate (RIVAS-MARTínEZ \& al., 2002). L. nobilis - a species which is notably sensitive to the rigidity of the winter climate, but also well to substantial water stress in the summer generally dominates woodlands located in inland areas of Thermo-Mesomediterranean thermotypes, with a subhumid ombrotype (mean annual rainfall: $700-800 \mathrm{~mm}$ ). More specifically, these woodland aspects can be observed on slopes of low inclination (Nature Reserve S. Ninfa, Menfi, Sciacca, Bisacquino, Buccheri), in moist depressions (Sambuca di Sicilia, Montemaggiore Belsito), along river (Bisacquino) or stream beds (Partanna, Bivona, Sambuca di Sicilia) or even in shaded ravines (M. Hyblaean near S. Andrea and Pietra di Valle; BRULLO \& al., 2001).

Laurel is known to prefer a certain soil coolness, although it avoids excessive water stagnation. It often lives on clay and flysh, near limestone outcrops, but sometimes also on gypseus and volcanites. In western and central
Sicily these mainly contain brown soil (Calcixerollic Xerochrepts), rather humid and rich in detritic materials (FIEROTTI \& al., 1988; FierotTI, 1997), associated with clay-rich soil.

In the southern area of the Sicani mountains, bay laurel woodlands shows a marked forest potential, suggesting the species might have been more widespread in the past, before the destruction of the original primary communities to obtain areas for cultivation.

Particularly interesting are the laurel aspects located in Contrada Menta (Sambuca di Sicilia), distributed in a wide area, close to limestone screes. Other similar aspects of the same vegetation were found in the peririparian area near Bivona (Torrente Alba), Partanna (Torrente Binaia in Contrada Stretto) and those located along carbon dorsals near Arancio Lake. These sites are characterized by very old L. nobilis stumps, showing signs of antique coppicing, with plenty of branches up to 13-15 metres high, and $30-45 \mathrm{~cm}$ in diameter. Individuals of bay laurel are in a good vegetative condition, with abundant fruiting, producing large quantity of seedlings in the undergrowth. Equally interesting are the aspects located in the Hyblaean area, with a continuous and dense structure and with very exuberant individuals (BRULLO \& al., 2001). 
Some other bay laurel woodlands monitored in Sicily occupy smaller surfaces, up to $250-400 \mathrm{~m}^{2}$. These are often scattered and degraded woodlands located in humid and shaded environments or on the edge of rocky outcrops, where they sometimes form luxuriant hedges, thereby enhancing the natural resources, the environment and the landscape, as well as being of use to fauna.

\section{VEGETATION SERIES AND CATENAL CONTACTS}

L. nobilis woodlands in Sicily often represent the most structured vegetation of an edaphichygrophilous series, ascribed to the Acantho mollis-Lauro nobilis sigmetum (Figure 2). This is dynamically related to mantle vegetation with Rubus ulmifolius (Roso sempervirentis-Rubetum ulmifolii), in which common species are Prunus spinosa, Pyrus amygdaliformis, Rosa canina, Clematis vitalba, Rosa sempervirens, etc. Close to rivers - often in high slope areas - bay laurel woodland appears at times with riparian vegetation of the series of Populus nigra and Salix pedicellata (Ulmo canescentis-Salico pedicellatae sigmetum).
On deeper soils with high clay content, L. nobilis woodland makes catenal contact with Quercus virgiliana sigmetum (Lauro-Querco virgiliane sigmetum), whose more structured aspect is represented by deciduous woodland of Lauro nobilisQuercetum virgilianae. This association, described for south-eastern Sicily (BRULLO \& al., 2001), was also found in some scattered areas of the Sicani mountains, impoverished by humans in order to obtain fields for cultivation (Figure. 2).

Along the screes located at the base of limestones reliefs, the Acantho mollis-Lauro nobilis sigmetum also shows catenal contact with the Quercus ilex vegetation series (Rhamno alaterniQuerco ilicis sigmetum). In the study area of the Sicani mountains, this latter series is represented by Quercus ilex woodland referred to Rhamno alaterni-Quercetum ilicis subass. pistacietosum terebinthi; thickets with a predominance of Pistacia terebinthus; Rhus coriaria shrublands; Ampelodesmos mauritanicus grassland (Helictotricho convoluti-Ampelodesmetum); and therophytic grassland referred to the alliance Trachynion distachyae.

Close to limestone cliffs, the laurel vegetation makes catenal contact with Rhamno alaterni-Eu-

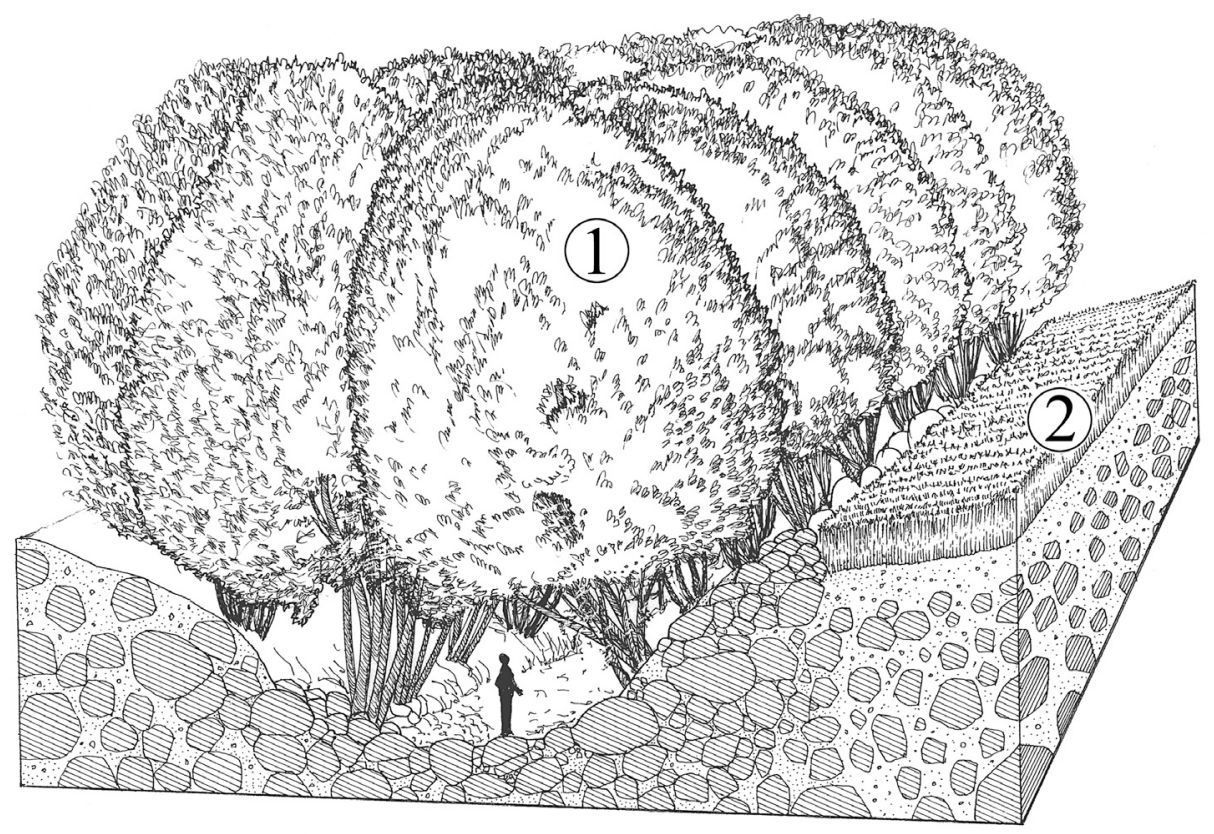

Figure 2.- Schematic block of vegetation: 1 - Laurus nobilis woodland (Acantho mollis-Lauretum nobilis); 2 Cultivated field weed communities (Legousio hybridae-Biforetum testiculatae). 
phorbio dendroidis euphorbietoso bivonae sigmetosum, located along xeric rocky outcrops, and represented by sclerophyllic and summer deciduous vegetation with Olea europaea var. sylvestris and Euphorbia dendroides (Rhamno alaterni-Euphorbietum dendroidis subass. euphorbietosum bivonae); Hyparrhenia hirta grassland (Hyparrhenietum hirto-pubescentis); and therophytic communities referred to the alliance Trachynion distachyae.

\section{Conservation ASPECTS}

Council Directive 92/43/EEC, whose aim is the conservation of species and habitats biodiversity in Europe, considers "Arborescent Matorral with Laurus nobilis" (5230) to be, a priority habitat of Community importance. In a recent study carried out by the ItALIAN Botanical Society (2002), only 25 sites were listed on the whole national territory: therefore the unpublished sites of the present paper serve as a contribution to the national list since they represent the best preserved and most extensive sites.

This vegetation is located in the Sicani mountains, and particularly in Sambuca di Sicilia (Contrada Menta) and Bivona (Torrente Alba). The first of these is included in Monte Genuardo and the Santa Maria del Bosco Nature Reserve, where one of the patches extends for almost one hectare (Figure 3). The second refers to another consistent laurel community extending for $6000 \mathrm{~m}^{2}$, locally known as "Viale degli Allori" (Laurel Avenue), which refers to dense and intricate vegetation located along a stream. In both cases these are residual woodlands with large polycormic L. nobilis individuals, 12-14 metres in height, fortunately saved from the intense transformation of the territory. Considering the singularity, rarity and environmental-naturalistic importance of the coenosis, these sites should be given more appropriate protection, starting with the sites not included in protected areas.

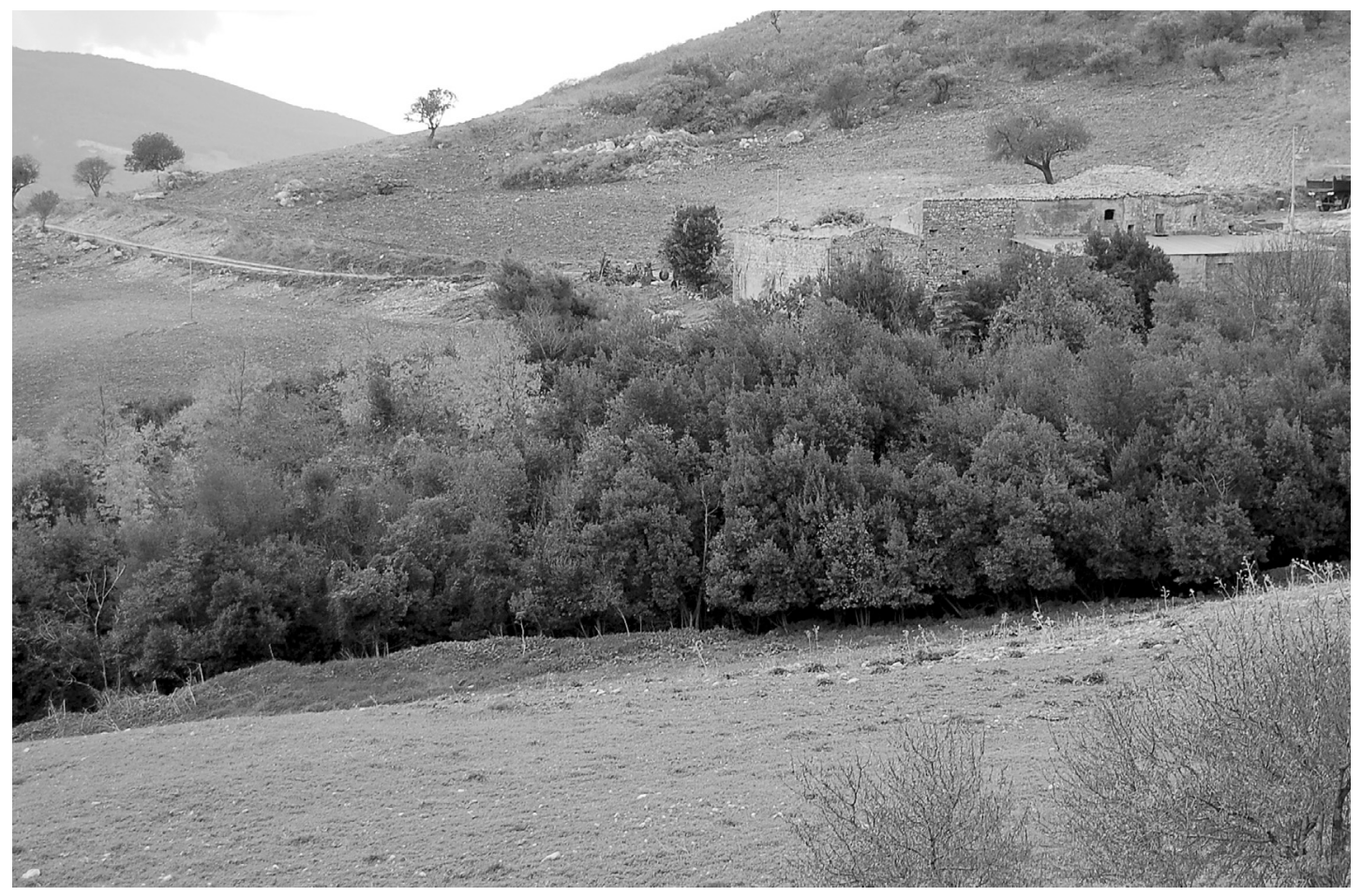

Figure 3.- Laurus nobilis woodland in "Monte Genuardo and S. Maria del Bosco Nature Reserve" (Sambuca di Sicilia). 
The main risks are related to this laurel vegetation's own ecology, which is closely dependent on a particular microclimate and hydrogeology of the same biotopes, and rapidly feel the effects of anthropic alterations (hydric regulation, lowering of water levels, etc.). It is also necessary to restrict coppicing and cutting, to provide continuous monitoring of the ecological factors, and eventually also a series of recovery and/or restoration measures in order to maintain this unique relict forest vegetation.

\section{CONCLUSIONS}

Laurophyll vegetation is typical of subtropical climates with low seasonality. This climate currently occurs in some areas of the world such as Japan, China, Eastern Australia, Florida, Canary Islands, Argentina and in Natal, South Africa (PIGNATTI \& NIMIS, 1995). Laurus nobilis woodlands in the Mediterranean region can be considered as "fragments" of vegetations widely occurring in warm and humid climate periods, isolated, as documented by the fossil record, some of them found in Sicily (BÉGuinot, 1929; GiACOBBE, 1939; ToRNABENE, 1859; PASTA, 2006). L. nobilis is indeed considered a residual element of an ancient Tertiary flora, re-adapted to the inside of the forest consortiums which colonized the territory after the Pleistocene climate changes (SCHMID, 1949; GIACOMini \& Fenaroli, 1958; Montelucci, 1977; Palamarev, 1989; Pignatti, 1995; Barbero \& Quezel, 1994; FilibeCK \& al., 2004; FilibeCK, 2006). The subsequent regression of this vegetation was presumably also caused by human activities such as deforestation and cultivation of the warm, moist and productive valley bottoms near humid zones, where relictual woodlands of L. nobilis can be found (GIANGUZZI, 2004).

The recognition of the Acantho mollis-Lauretum nobilis in Sicily is of particular phytogeographic interest, as well has having certain naturalistic-environmental value. The association interpreted as a southern vicariant of other coeno-

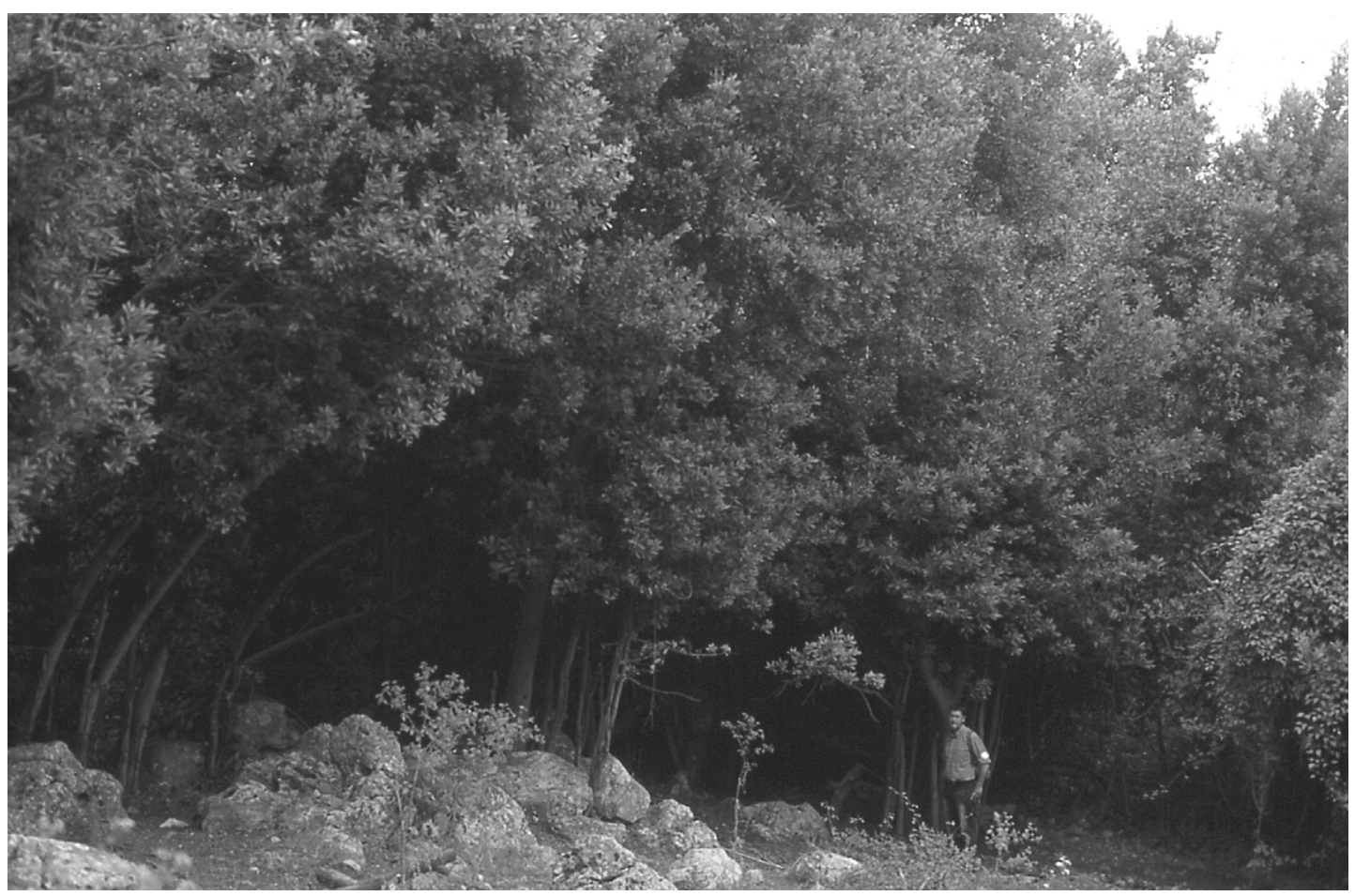

Figure 4.-Aspects of Acantho mollis-Lauretum nobilis in the Sicani mountains. 
ses occurring in the Mediterranean area serves to increase the variability of the woody formations known so far, and also, supplies useful elements for the understanding the recent paleoenvironmental dynamic of the insular territory.

In accordance with RODRÍGUEZ GUITIÁN \& al. (2007), referring to laurophyllous vegetation in the Iberian peninsula, in Sicily the distributional pattern of these patches seems to be indicative of refugia sites. That is to say, its floristic component, with low cold tolerance, was isolated during the most critical phases of the Pleistocene and Holocene.

An unresolved issue seems to be the syntaxonomic classification of Laurus nobilis vegetation, both at the alliance and at the order level. In fact, while some authors refer the different coenoses to the Arbuto unedonis-Laurion nobilis (Pistacio lentisci-Rhamnetalia alaterni), others consider it more appropriate to include the aspects from the Italian peninsula in the order Quercetalia ilicis
(AllegrezZA \& al., 2006; BACCHETTA \& al., 2007; BIONDI \& al., 2002, 2004).

From the viewpoint of nature conservation, the sites in the study tend to be in a rather vulnerable state, although they often show a high capacity of renewal from seedlings. Considering the scientific-naturalistic relevance of the formation and its status of priority habitat, a more suitable protection of the residual populations would appear to be necessary.

\section{ACKNOWLEDGMENTS}

This work was carried out with approx. $60 \%$ of M.I.U.R. contribution (Resp. Prof. L. Gianguzzi). The authors wish to thank: Prof. Jesús Izco and two anonynous referees, for their valuable suggestions, Giulia Casamento, Carolina Di Patti, Orazio Caldarella, Alessandro Gristina, Gaspare Incardona, Paola Lazzara and Giuseppe Ferlita, for their useful support during our field work.

\section{REFERENCES}

Álvarez Arbesú, R. -2005- La cubierta vegetal de los acantilados asturianos - Publ. Dpto. Biol. Org. Sists. Univ. Oviedo, 199 pp.

Albo, G. - 1919 - La vita delle piante vascolari nella Sicilia Meridionale-Orientale. Parte II: Flora - Ragusa, pp. 1-308.

Allegrezza, M., Biondi, E. \& Felici, S. -2006- A phytosociological analysis of the vegetation of the central sector of the adriatic aspect of the Italian peninsula Hacquetia 5 (2): 5-45.

Agnesi, V., Di Maggio, C., Macaluso, T., Masini, F., Petruso, D. \& Simonelli, C. - 2000-Quaternary Environmental-climatic changes in Sicily - Mem. Soc. Geol. It. 55: 339-344.

Anzalone, B., Lattanzi, E., Lucchese, F. \& Padula, M. 1997- Flora vascolare del Parco Nazionale del Circeo (Lazio) - Webbia 51 (2): 251-341.

Arkman, Y., Barbero, M. \& Quezel P. -1978 - Contribution à l'étude de la végétation forestière d'Anatolie méditerranéenne - Phytocoenologia 5: 1-79.

Arkman, Y., Quezel, P., Ketenoglu, O. \& Kurt I. -1993Analyse syntaxonomique des forêts de Liquidambar orientalis en Turquie - Ecol. Medit. 19: 49-57.

Bacchetta, G., Farris, E., Fenu, G., Filigheddu, R., Mattana, E. \& Mulè, P. - 2007- Contributo alla conoscenza dei boschi a Laurus nobilis L. della Sardegna, habitat prioritario ai sensi della Direttiva 92/43/CEE - Fitosociologia 44 (2): 239-244.
Barbero, M., Benadid, A., Peyre, C., Quézel, P. -1981Sur la presence au Maroc de Laurus azorica (Seub.) Franco - An. Jard. Bot. Madrid 37: 467-472.

Barbero, M. \& Quézel,P. - 1978 - Les groupements forestières de Grece Centro-Méridionale - Ecol. Medit., 2: 3-79.

Barbero, M. \& Quézel, P. - 1980 - La végétation forestière de Crète - Ecol. Medit. 5: 175-210.

Barbero, M. \& Quezel, P. - 1994 - Place, role et valeur historique des éléments laurifoliés dans les végétations préforestières oust-méditerranéenne - Ann. Bot. (Roma) 52: 81-131.

Barbero, M., Quézel, P. \& Rivas-Martínez, S. -1981Contribution a l'etude des groupements forestières et préforestières du Maroc - Phytocoenologia 9: 311-412.

Bartolo, G., Brullo, S., Minissale, P. \& Spampinato, G. -1990aContributo alla conoscenza dei boschi a Quercus ilex della Sicilia - Acta Bot. Malacitana 15: 203-215. Malaga.

Bartolo, G., Brullo, S., Minissale, P. \& Spampinato, G. 1990b - Flora e vegetazione dell'Isola di Lampedusa Boll. Acc. Gioenia Sci. Nat. 21(334): 119-255.

Béguinot, A. - 1929- Illustrazione delle filliti quaternarie dei travertini palermitani conservate nel Museo di Geologia della R. Università di Palermo - Arch. Bot. 5: 143-173.

Biondi, E. — 1972 - Stazioni di Laurus nobilis L. nelle Marche - Arch. Bot. Biogeogr. Ital. 48, ser. 4, 17 (1-2): 74-79.

Biondi, E. -1982 - L'Ostrya carpinifolia Scop. sul litorale delle Marche (Italia centrale) - Studia Geobot. 2: 141-147. 
Biondi, E., Gigante, D., Pignattelli, S. \& Venanzoni, R. 2002 - I boschi del piano collinare della Provincia di Terni - Fitosociologia 39 (1): 135-160.

Bolòs, O., De Masalles, R.M., Ninot, J. M. \& Vigo, J. 1996- A survey on the vegetation of Cephalonia (Ionian islands) - Phytocoenologia $26: 81-123$.

Bonomo, R., Raimondo, F. M., Castiglia, G. \& Lentini, F. - 1977/78 - Aspetti di vegetazione palustre, prativa e forestale in località "Salaci" di Cammarata con riferimento alla florula medicinale - Atti Accad. Sci. Palermo 4, 37 (1): 3-50.

Braun-Blanquet, J. - 1932 - Plant sociology, 1st ed McGraw-Hill Book Company, New York and London.

Brullo, S., Costanzo, E. \& Tomaselli, V. -2001 - Etude phytosociologique sur les peuplements à Laurus nobilis dans les Monts Iblei (Sicile sud-orientale) - Phytocoenologia 31 (2): 249-270.

Brullo, S., Gianguzzi, L., Ilardi, V. \& Salmeri, C. - 2002Matorral di Laurus nobilis (5230) in Sicilia. - In Società Botanica Italiana, Ricerca sugli habitat prioritari presenti in Italia (III fase). Minist. Amb., Serv. Conserv. Natur. Roma.

Brullo, S. \& Guarino, R. - 1998 - The forest vegetation from the Garda lake (N Italy) - Phytocoenologia 28(3): 319-355.

Brullo, S. \& Marcenò, C. - 1985- Contributo alla conoscenza della classe Quercetea ilicis in Sicilia - Not. Fitosoc. 19: 183-229.

Brullo, S. \& Spampinato, G. - 1990 - La vegetazione dei corsi d'acqua della Sicilia - Boll. Acc. Gioenia Sci. Nat. 23: 183-229.

Bueno Sánchez, A. \& Fernández Prieto, J. A. -1991 Acebuchales y lauredales de la costa cantabrica - Lazaroa 12: 273-301.

Castroviejo, S. \& als. (Eds.) - 1986/2003 - Flora iberica. Plantas vasculares de la Península Ibérica e Islas Balearica - Real Jardin Botanico, C.S.I.C., Madrid.

Chiappini, M. - 1960 - Nuove stazioni di "Laurus nobilis" L. nella Sardegna Nord-Occidentale - Webbia 15: 347-391.

Conti, F., Abbate, G., Alessandrini, A., Blasi, C. (Ed.) 2005- An annotated Checklist of the Italian Vascular Flora - Ministero dell'Ambiente e della Tutela del Territorio (Direzione per la Protezione della Natura) e Dipartimento di Biologia Vegetale dell'Università degli Studi di Roma "La Sapienza". Palombi Editori, p. 420.

Deil, U. - 1994- Felsgesellschaften beiderseits der Strasse von Gibraltar - Hoppea, Denkschr. Regensb. Bot. Ges. 55: 757-814.

Desole, L. - 1947 - Diffusione e localizzazione della macchia-foresta a base di Laurus nobilis L. nella Sardegna settentrionale - Bull. Ist. Bot. Univ. Sassari 3.

Desole, L. - 1949 - Diffusione e localizzazione della macchia foresta a base di Laurus nobilis L. nella Sardegna settentrionale (cont.). Stud. Sassar., sez. 2, 27: 3-24.

Díaz González, T. E. \& Fernandez Prieto, J. A. -1994La vegetation de Asturias - Itinera Geobot. 8: 243-528.

Fenaroli, L. \& Gambi, G. - 1976- Alberi: dendroflora italica - Trento.
Ferguson, D.K. - 1974 - On the taxonomy recent and fossil species of Laurus (Lauraceae) - Bot. J. Linn. Soc. 68: 51-72.

Fierotti, G. - 1997 - I suoli della Sicilia con elementi di genesi, classificazione, cartografia e valutazione dei suoli - Dario Flaccovio, Palermo, 359 pp.

Fierotti, G., Dazzi, C. \& Raimondi, S. - 1988- Commento alla Carta dei Suoli della Sicilia - Regione Siciliana, Assessorato Territorio e Ambiente Palermo, 19 pp.

Filibeck, G. - 2006- Notes on the distribution of Laurus nobilis L. (Lauraceae) in Italy - Webbia 61 (1): 45-56.

Filibeck, G., Arrigoni, P.V. \& Blasi, C. -2004- Some phytogeographical remarks on the forest vegetation of Colchis (Western Georgia) - Webbia 59 (1): 33-82.

Giaccobbe, A. -1939 - Ricerche geografiche ed ecologiche sul Laurus nobilis L. - Arch. Bot. It. 15: 33-82.

Giacomini, V. \& Zaniboni, A. - 1946- Osservazioni sulla variabilità del "Laurus nobilis L." nel bacino del Lago di Garda - Arch. Bot. 22 (1-4): 1-16.

Giacomini, V. - 1969 - Il paesaggio geobotanico del Lago di Garda con un saggio di bibliografia naturalistica benacense. Il Lago di Garda - Atti del Conv. Intern. de1l'Ateneo di Salò "Il lago di Garda: storia di una comunità lacuale": 100-104.

Giacomini, V. \& Fenaroli, L. - 1958 - La flora. Conosci l'Italia - Touring Club Italiano, Milano, Milano 2: 1272.

Gianguzzi, L. (a cura di) - 2004- Il paesaggio vegetale della Riserva Naturale Orientata "Bosco della Ficuzza, Rocca Busambra, Bosco del Cappelliere, Gorgo del Drago" - Collana Sicilia Foreste (22). Azienda Foreste Demaniali della Regione Siciliana, Palermo pp.160.

Gianguzzi, L. - 1999- Flora e vegetazione dei Nebrodi. Itinerari didattici - Ass. Agric. For. Reg. Siciliana, 231 pp.

Gianguzzi, L., Ilardi, V. \& Raimondo, F.M. -1996- La vegetazione del promontorio di Monte Pellegrino (Palermo) - Quad. Bot. Amb. Appl. 4 (1993): 79-137.

Gianguzzi, L \& La Mantia, A. -2008 - Contributo alla conoscenza della vegetazione e del paesaggio vegetale della Riserva Naturale "Monte Cofano" (Sicilia occidentale) (con allegata Carta sinfitosociologica della vegetazione, scala 1:20.000) - Fitosociologia 45 (1) suppl. 1: $1-55$.

Gianguzzi, L., Raimondo, F.M. \& Riggio, S. -1995- Relics of riverine Platanus orientalis L. forest in the Oreto valley, Palermo - Giorn. Bot. Ital. 129 (2): 187.

Gianguzzi, L., Romano, S., D'Amico, A. \& Incardona, G. -2007- Caratterizzazione fitosociologica di alcuni nuclei residuali di laureto sui Monti Sicani (Sicilia centro-occidentale) - Atti $102^{\circ}$ Congr. Soc.i Bot. Italiana. Palermo 26-29 Settembre 2007.

Grimaldi, G. \& Scaletta, C. -1997 - L'insediamento neolitico di Contrada Stretto. Caratteristiche geomorfologiche e ipotesi ricostruttive. In: Balistreri, A., Giannitrapani, E., Nicoletti, F., Picciotto, C., Titi, L., Tusa, S. (Eds.). Prima Sicilia. Alle origini della società siciliana. Regione Siciliana. Pp. 213-222. Assess. Ben. Cult., Amb. Pubbl. Istruz. 
Guarino, R. - 1998 - La vegetazione dei Monti Peloritani (Sicilia nord-orientale) - Mem. Doc. (inéd.). Dip. Botanica, Univ. Stud. Catania, $301 \mathrm{pp}$.

Gussone, G. - 1842-44 - Flora siculae Synopsis - Ex Regia Typografia, Neapoli, pp. 647, Add. pp. 883.

Heywood, V.H. (ed.) - 1993 - Flowering plants of the world. - B T Batsford Ltd, London.

Honrado, J., Alves, P., Alves, H.N. \& Barreto Caldas, F. 2003 - A vegetaçao do Alto Minho. Esboço Fitossociológico da Vegetaçao Natural do Extremo Noroeste de Portugal (Sectores Galaico-Português e Geresiano) Quercetea 5: 3-102.

Jalas, J. \& Suominen, J. (Eds.) - 1991 - Atlas Florae Europaeae. Distribution of vascular plants in Europe. Vol. 9 (Paeoniaceae to Capparaceae) - Publ. Soc. Biol. Fennica, Helsinki, 110 pp.

Karpati, I. \& Karpati, V. -1861 - Die zönologischen Verhältnisse Auenwälder - Acta Bot. Acad. Sci. Hung. 7: 235-301.

Knapp, R. - 1965 - Die Vegetation von Kephallinia, Griechenland - Verlag Otto Koeltz, Loenigstein, 206 pp.

Komarov, L. (Ed.) -1937- Flora SSSR, Vol. 7 - Mosca Leningrado.

Leporatti, M. L., Lattanzi, E. -1996 - Furbara e Macchiatonda. - In Dinelli, A. \& Guerriera, P.M. (Eds.). Ambienti di particolare interesse naturalistico del Lazio. Regione Lazio. Pp. 74-75. - Assess. Cult. Dip. Biol. Veg. Univ. La Sapienza. Roma.

Loidi, J., García-Mijangos, I., Berastegui, A. \& Darquistade, A. - 1997- Nuevos datos sobre los bosques secundarios (prebosques) del sector Cántabro-Euskaldún - Lazaroa 18: 165-172.

Lojacono, M. -1888-1909- Flora Sicula, o Descrizione delle Piante vascolari spontanee o indigenate in Sicilia, 3 vols. - Tip. Virzì., Palermo.

Lucchese, F. \& Pignatti, S. - 1990 - Sguardo sulla vegetazione del Lazio Marittimo - Quad. Acc. Naz. Lincei 264: 5-48

Lucchese, F. - 1992 - La flora della Riserva Naturale di Palo Laziale (Roma) - Ann. Bot. 48 (7): 263-289.

Lucchese, F. - 1996- Sughereta di Fossanova (M. Musoni) - In: Dinelli, A. \& Guerriera, P.M. (Eds.). Ambienti di particolare interesse naturalistico del Lazio. Regione Lazio. Pp . 286-288. Assess. Cult./ Dip. Biol. Veg. Univ. La Sapienza. Roma.

Marcenò, C., Gristina, A. \& Scuderi, L. -2007- Nuovi dati distributivi relativi a specie di particolare interesse rinvenute lungo il bacino del Belice ed in aree limitrofe - Atti $102^{\circ}$ Congr. Soc. Bot. Italiana. Riass. Palermo 26-29 Settembre 2007.

Mabberley, D.J. - 1997 - The plant-book: a portable dictionary o the vascular plants, 2nd ed. - Cambridge Univ. Press., Cambridge.

Meusel, H., Jäger, E. \& Weinert, E. -1965- Vergleichende Chorologie der Zentraleuropaischen Flora - G. Fischer, Jena.

Montelucci, G. - 1977 - Lineamenti della vegetazione del Lazio - Ann. Bot. 35-36: 1-108.
Montelucci, G. -1946- Investigazioni botaniche nel Lazio. II, - Nuove stazioni laziali di "Laurus nobilis L." - Arch. Bot. 22: 44-51.

Montelucci, G. - 1964- Ricerche sulla vegetazione de1l'Etruria XIII. Materiali per la flora e la vegetazione di Viareggio - Webbia 19 (1): 73-347.

Moragues, J.R. \& Moragues, E. -2004- Les comunitats de llorers (Laurus nobilis) als torrents de la Serra de Tramuntana de Mallorca, une reliquies del passat - IV journ. Med. Amb. Illes Balears, 16pp.

Orsomando, E., Catorci, A. \& Cenci, C. A. -1992- Aspetti fitogeografici ed ecologici del bosso (Buxus sempervirens L.) in Umbria - Stud. inform. IRRES: 85-104.

Palamarev, E. - 1989- Paleobotanical evidences of the Tertiary history and origin of the Mediterranean sclerophyll dendroflora - Plant Syst. Evol. 162: 93-107.

Pasta, S. - 2001 - Contributi alla conoscenza botanica delle isole minori circumsiciliane. I. Sintesi aggiornata delle conoscenze botaniche sull'Isola di Lampedusa finalizzata alla conservazione delle sue emergenze floristico-vegetazionali - Naturalista sicil. s. IV, XXV (suppl.): 19-70.

Pasta, S. -2006 - I fossili vegetali. - In A.VV., Il travertino di Alcamo. Proposta per l'istituzione di un geosito. Regione Siciliana, Assessorato Regionale BB.CC.AA. e P.I., Palermo, pp. 29-30.

Pasta, S. \& La Mantia, T. -2006- Lineamenti sulla flora e la vegetazione dell'area della Riserva Naturale "Grotta di Santa Ninfa - In: Casamento, G. (Ed.). Le riserve naturali siciliane gestite da legambiente: un contributo alla conoscenza e alla tutela. Natur. sicil. s. 4, 25 (suppl.): 299-323.

Pavari,A. - 1933 - Castelusano: il grande Parco dell'Urbe - L'Alpe 20: 297-310.

Pedrotti, F. \& Gafta, D. - 1992- Tipificazione di tre nuove associazioni forestali ripariali nell'Italia meridionale Doc. Phytosoc. n.s. 14: 557-560.

Pedrotti, F. - 1993 - La vegetazione forestale italiana Atti dei Convegni Lincei 115: 39-78.

Pignatti, S. - 1976 - Geobotanica. In: Cappelletti, C.(Ed.). Trattato di Botanica (terza edizione). Pp. 801-997 UTET Torino 2.

Pignatti, S. - 1978 - Dieci anni di cartografia floristica ne11'Italia di Nord-Est - Inform. Bot. Ital. 10: 212-217.

Pignatti, S. - 1979- I piani di vegetazione in Italia Giorn. Bot. Ital. 113 (5-6): 411-428.

Pignatti, S. - 1982- Flora d'Italia, 3 Vols. - Edagricole. Bologna.

Pignatti, S. - 1995 - Ecologia vegetale - UTET, Torino, pp. 532.

Pignatti, S. \& Nimis, P. L. - 1995 - Biomi - In: Pignatti S. (Ed.). Ecologia vegetale, pp. 319-355 - UTET, Torino.

Pirone, G. - 2000 - La vegetazione ripariale nei versanti nord-orientali del Gran Sasso d'Italia e dei Monti della Laga (Abruzzo, Italia) - Fitosociologia 37 (2): 65-86.

Raimondo, F.M., Gianguzzi L. \& Schicchi, R. - 1994Carta della vegetazione del massiccio carbonatico delle Madonie (Sicilia centro-settentrionale) - Quad. Bot. Ambientale Appl., 3 (1992): 23-40. 
Raimondo, F.M., Mazzola, P. \& Castiglia, C. - 1980Lembi relitti di macchia-foresta a Laurus nobilis L. sulle Madonie (Sicilia) - Giorn. Bot. Ital., 114 (3- 4): 137.

Raven, P.H. - 1973 - The evolution of Mediterranean Floras. In: Di Castri F. \& Mooney H. A. (Eds.). Mediterranean type ecosystems, Ecological studies Springer-Verlag 1973.

Rivas-Martínez, S., Diaz, T. E., Fernàndez-Gonzàlez, F., Izco, J., Lousa, M., Penas, A. -2002- Vascular plant communities of Spain and Portugal. Addenda to the syntaxonomical checklist of 2001 - Itinera Geobot. 15: 5-922.

Rivas-Martínez, S. \& Loidi, J. - 1988 - Los robledales mesofiticos navarro-alaveses (Crataego laevigataeQuercetum roboris) - Lazaroa 10: 81-88.

Rodríguez Guitián, M.A., Romero Franco, R. \& Ramil Rego, P. - 2007- Caracterización ecológica y florística de las comunidades lauroides del occidente de la Cornisa Cantábrica (Noroeste ibérico) - Lazaroa 28: 35-65 (2007).

Schmid, E. - 1949 - Prinzipien der naturlichen Gliederung der Vegetation des Mediterrangebietes (mit einer halbschematischen Karte) - Ber. Schweiz. Bot. Ges. 19: 169-200.

Scuderi, L. - 2006- Flora e vegetazione della provincia di Trapani (Sicilia) - Mem. Doc. (inéd.). Dip.Botanica, Univ. Stud. Catania. 542 pp.
Società Botanica Italiana -2002- Ricerca sugli habitat prioritari presenti in Italia (III fase): Matorral di Laurus nobilis (5230) - Minist. Amb., Serv. Cons. Natur., Roma.

Spada, F. - 1977- Primi lineamenti della vegetazione del comprensorio tolfetano-cerite - Quad. Acc. Naz. Linc. 227: $37-50$.

Stace, C. - 1991 - New Flora of the British Isles. - Cambridge Univ. Press.

Tonelli, V. - 1996 - "Antica Lavinium" Pratica di Mare In: Dinelli, A. \& Guerriera, P.M. (Eds.). Ambienti di particolare interesse naturalistico del Lazio. Regione Lazio. Pp. 190-192 - Asses. Cult. Dip. Di Biol. Veg. Univ. La Sapienza., Roma.

Tornabene, F. -1860- Flora fossile dell'Etna - Atti Accad. Gioenia Sci. Nat. Catania s. 2, 16:1-147.

Tutin, T. G., \& als. (Eds.) -1964/1980- Flora Europaea 5 vols. - Cambridge Univ. Press., Cambridge.

Ubaldi, D. -1988- La vegetazione boschiva della provincia di Pesaro e Urbino - Esercitaz. Acc. Agr. Pesaro 20: 99192.

Ubaldi, D., Puppi, G., Speranza, M. \& Zanotti, A.L., -1984Primi risultati sulla tipologia fitosociologica dei boschi di Quercus pubescens della provincia di Pesaro e Urbino - Arch. Bot. Biogeogr. Ital. 60: 150-168.

Zohary, M. -1960- The maquis of Quercus calliprinos in Israel and Jordan - Bull. Res. Counc. Israel 9D: 51-72.

Recibido: 30 mayo 2008

Aceptado: 11 enero 2010

\section{APPENDIX \\ List of syntaxa cited in the text}

Calluno vulgaris-Lauretum nobilis F. Prieto, Arbesú \& Bueno in T.E. Díaz \& F. Prieto 1994; Blechno spicant-Quercetum roboris Tüxen \& Oberdorfer 1958 subass. lauretosum nobilis Losa Quintana ex Izco, Amigo \& Guitian 1990; Celtido australis-Lauretum nobilis Bacchetta, Farris, Fenu, Filigheddu, Mattana \& Mulè 2007; Clematido cirrhosae-Quercenion ilicis Bacchetta, Bagella, Biondi, Farris, Filigheddu \& Mossa 2004; Fraxino orni-Lauretum nobilis Allegrezza, Biondi \& Felici 2006; Fraxino orni-Quercion ilicis Biondi, Casavecchia \& Gigante 2003; Hedero helicis-Lauretum nobilis Bueno Sànchez \& F. Prieto 1991 subass. lauretosum nobilis, subass. euphorbietosum amygdaloidis T.E. Díaz \& F. Prieto 1994, subass. davallietosum canariensis Deil 1994; Helictotricho convoluti-Ampelodesmetum mauritanici Minissale 1994; Hyparrhenietum hirto-pubescentis A.\& O. Bolòs \& Br.-Bl. in A. \& O. Bolòs 1950; Lauro-Quercetum virgilianae Brullo, Costanzo \& Tomaselli 2001; Legousio hybridae-biforetum testiculatae Di Martino \& Raimondo 1976; Myrto communis-Salicetum atrocinereae Biondi \& Bagella 2006 subass. lauretosum nobilis Bacchetta, Farris, Fenu, Filigheddu, Mattana \& Mulè 2007; Rhamno alaterni-Euphorbietum dendroidis (Trinajstic 1973) Géhu \& Biondi 1997 subass. euphorbietosum bivonae (Gianguzzi, Ilardi \& Raimondo 1996) Gianguzzi \& La Mantia 2007; Ornithogalo pyrenaici-Quercetum ichnusae Bacchetta, Biondi, Farris, Filigheddu \& Mossa 2004 subass. lauretosum nobilis Bacchetta, Farris, Fenu, Filigheddu, Mattana \& Mulè 2007; Rusco aculeate-Quercetum ilicis Biondi, Gigante, Pignattelli \& Venanzoni 2002; Omphalodo nitidae-Lauretum nobilis Honrado, P. Alves \& F.B. Caldas 2003; Rhamno alaterni-Quercetum ilicis Brullo \& Marcenò 1985 subass. pistacietosum terebinthi Gianguzzi, Ilardi \& Raimondo 1996; Roso sempervirentis-Rubetum ulmifolii Blasi, Cutini, Di Pietro \& Fortini 2001; Tamo communis-Lauretum nobilis Honrado, P. Alves \& F.B. Caldas 2003; Ulmo canescentis-Salicetum pedicellatae Brullo \& Spampinato 1990. 